Meta

Journal des traducteurs

Translators' Journal

\title{
Les dénominations de la règle à calcul
}

\section{Claude Pierre Boisson}

Volume 41, numéro 4, décembre 1996

La dénomination

URI : https://id.erudit.org/iderudit/002095ar

DOI : https://doi.org/10.7202/002095ar

Aller au sommaire du numéro

Éditeur(s)

Les Presses de l'Université de Montréal

ISSN

0026-0452 (imprimé)

1492-1421 (numérique)

Découvrir la revue

Citer cet article

Boisson, C. P. (1996). Les dénominations de la règle à calcul. Meta, 41(4),

525-566. https://doi.org/10.7202/002095ar
Résumé de l'article

L'histoire de la règle à calcul est reprise d'une manière systématique, et sa dénomination est analysée dans 41 langues. Une comparaison de ces termes nous permet de postuler pour $\mathrm{r}$ objet en question un "schéma définitionnel" développé, de nature panlinguistique, qui pourrait fournir une approximation empirique pour une analyse du concept / règle à calcul /. Dans ce scheme, chaque langue sélectionne typiquement deux éléments notionnels, de sorte que ces termes ressemblent à des définitions elliptiques de l'objet, certaines dénominations étant favorisées pour diverses raisons qui ne sont pas nécessairement cognitives ou linguistiques. 


\title{
LES DÉNOMINATIONS DE LA RÈGLE À CALCUL
}

\author{
Claude Pierre Boisson \\ Centre de Recherche en Terminologie et Traduction, Université Lumière, Lyon, France
}

\begin{abstract}
Résumé
L' histoire de la règle à calcul est reprise d'une manière systématique, et sa dénomination est analysée dans 41 langues. Une comparaison de ces termes nous permet de postuler pour l'objet en question un «schéma définitionnel» développé, de nature panlinguistique, qui pourrait fournir une approximation empirique pour une analyse du concept / règle à calcull. Dans ce schème, chaque langue sélectionne typiquement deux éléments notionnels, de sorte que ces termes ressemblent à des définitions elliptiques de l'objet, certaines dénominations étant favorisées pour diverses raisons qui ne sont pas nécessairement cognitives ou linguistiques.
\end{abstract}

\begin{abstract}
The history of the slide-rule is retold in a systematic way, and its denomination is analysed in 41 languages. A comparison of these terms allows us to postulate for the object an extensive panlinguistic "definition scheme", which might provide an empirical approximation to an analysis of the concept / slide-rule 1. Each language typically selects from this scheme a couple of notional elements, so that these terms look like elliptical definitions of the object, some denominations being favoured for a number of reasons which are not necessarily cognitive or linguistic.
\end{abstract}

On enregistrera avec le temps et mettra en dictionnaires et en grammaires toutes les langues de l'univers, et on les comparera entre elles; ce qui aura des usages très grands tant pour la connaissance des choses, puisque les noms souvent répondent à leurs propriétés (comme l'on voit par les dénominations des plantes chez de différents peuples) que pour la connaissance de notre esprit et de la merveilleuse variété de ses opérations. Leibniz (1765, 1990: livre III, chap. IX, 264)

\section{A. INTRODUCTION}

Ceci est un exercice de «terminologie comparée» ou «terminologie multilingue» (Thoiron 1994), qui confronte diverses langues quant à leur manière de dénommer un objet bien défini. Il permettra de montrer concrètement à quels problèmes les utilisateurs des langues sont confrontés dans l'acte de dénomination. Il touchera aussi bien à la linguistique qu'à la technologie (dans l'acception rigoureuse mais quasiment disparue d'《étude de la technique», et non de «technique», comme couramment), et même un peu à la psychologie, et je ne chercherai pas à battre ma coulpe pour ce mixte impur mais réaliste.

Bernard Pottier a heureusement montré avec une série d'exemples devenus classiques comment chaque langue désigne le «pompier» en sélectionnant certains traits (la nature de ces traits reste à déterminer, nous en reparlerons tout à la fin). Citons-le 
(Pottier 1992a: 202): «Il convient de poser un modèle relationnel, duquel on pourra dériver les composés $[\ldots]$ dans différentes langues:

\begin{tabular}{|c|c|c|c|}
\hline$\underset{1}{\text { [quelqu'un }}$ & $\begin{array}{l}\text { lutter contre/éteindre } \\
2\end{array}$ & $\begin{array}{l}\text { feu } \\
3\end{array}$ & $\begin{array}{l}\text { (avec) pompe] } \\
4\end{array}$ \\
\hline $3-2-1:$ & $\begin{array}{l}\text { all. Feuerwehrmann } \\
\text { angl. firefighter }\end{array}$ & & \\
\hline $3-1:$ & angl. fireman & & \\
\hline 4-1: & $\begin{array}{l}\text { fr. pompier } \\
\text { esp. bombero }\end{array}$ & & \\
\hline $2-1:$ & fr. éteigneur (TL & )》. & \\
\hline
\end{tabular}

On pourrait ajouter l'arabe pour le choix 2-1, puisque pompier s'y dit «homme d'extinction», ou «éteigneur» (information de Hassan Hamze). Le russe, le tchèque et le polonais rentrent dans le schéma 2-1, l'italien dans le schéma 4-1. L'espéranto a plusieurs possibilités (Wells 1969), dont l'une, fajr-estingisto, réalise le schéma 3-2-1 sous une forme particulièrement claire.

On observera que le «modèle relationnel» est bel et bien une proposition et que c'est à partir de cette proposition que, par ellipse, vont se créer les dénominations, lesquelles garderont à l'état vestigial un certain caractère propositionnel, pourrait-on soutenir. D'ailleurs, Pottier dit cursivement à propos de ce recodage compact (pp. 79, 176): «Le "nom" n'est au fond qu'une proposition intégrée [...]. D'ailleurs, l'histoire même de nombreux substantifs montre qu'ils proviennent d'énoncés intégrés (cf. dérivation, composition)». Plus précisément, en effet, le «modèle relationnel» n'est autre qu'une partie de définition. On peut ainsi définir provisoirement le pompier de la sorte: «Un pompier est une personne telle que [cette personne éteint le feu avec une pompe]». C'est pourquoi je m'autoriserai à renommer le «modèle relationnel» de Pottier: «schéma définitionnel».

On peut aller un peu plus loin. En effet, noter que l'italien connaît aussi vigile del fuoco. Synchroniquement, vigile correspond à notre agent (personne appartenant à un corps spécial), d'où vigile (urbano) = agent de police. Si bien que vigile del fuoco correspond approximativement au schéma 3-1, comme l'anglais fireman, à ceci près toutefois que vigile désigne plus précisément l'appartenance à un corps spécialisé dans une fonction. De même, une variante espéranto est fajr-brigadano, où -an- est un suffixe indiquant le membre d'un groupe, donc à gloser «membre d'une brigade [s'occupant d'éteindre] les incendies». Ceci nous conduit à expliciter ct à enrichir le schéma de Pottier, car au lieu de quelqu' un pour 1, il convient de faire figurer agent («individu appartenant à un corps officiellement spécialisé dans une fonction»), sinon toute personne se trouvant là par hasard et contribuant à l'extinction du feu serait un «pompier»; or si l'on peut être pompier volontaire, donc concurremment à d'autres activités, on n'est pas «pompier» d'occasion, le suffixe -ier excluant le fortuit. On voit donc que la détermination du ou des schéma(s) définitionnel(s) exige prudence et collecte de matériaux maximalement divers pour être nourrie empiriquement. Ceci nous servira de leçon et d'inspiration pour la suite.

On observera aussi que le français éteigneur, et même à la limite l'anglais firefighter, prêtent à interprétation ambiguë, car au vu du suffixe -er, avons-nous à faire à une chose, à un instrument (à comparer à l'avion appelé jet fighter), ou bien s'agit-il d'un homme, d'un agent? D'autant que l'anglais a aussi fire-extinguisher pour l'actuel extincteur du français, terme qui aurait fort bien pu désigner un pompier. L'espéranto lève élégamment l'ambiguïté grâce à des suffixes caractéristiques de la chose (-il-) ou de l'agent humain (-ist-), et distingue donc régulièrement fajr-estingilo (extincteur) et 
fajr-estingisto (pompier). Dans cet ordre d'idées, on observera que le français peut avoir recours à des suffixes féminins pour les instruments, ce qui autorise des oppositions avec les agents humains: moissonneuse vs. moissonneur; fraiseuse vs. fraiseur; ponceuse, cireuse vs. ponceur, cireur; calculatrice, calculette, vs. calculateur (inspiré d'une remarque de François Lo Jacomo). Ceci peut éventuellement s'expliquer par un symbolisme latent, ou simplement par le fait que les noms d'agent étant normalement masculins en français, le contraste le plus économique est de créer un féminin sur le même suffixe.

Sur des questions connexes, consulter l'article de Talmy (1985), qui étudie le codage des mouvements dans différentes langues, partant d'un scénario et de la conflation de certains éléments du scénario dans la lexicalisation sous la forme d'un verbe de mouvement.

De manière complètement indépendante, dans un passage d'un article récent, Philippe Thoiron s'était intéressé brièvement à la dénomination de la règle à calcul en français et en anglais, commentant les faits dans une perspective voisine de celle de Pottier et ajoutant une série d'autres termes (Thoiron 1994; voir aussi Thoiron et alii, dans ce volume); c'est cet exemple et l'article de Thoiron qui constituent le germe de la présente monographie.

La règle à calcul est en effet un objet de choix pour ce genre d'études. Il a longtemps été l'emblème de l'ingénieur et de la société qu'il incarne (voir plus bas pour une des dénominations de l'arabe), suscitant parfois des réactions hostiles. Pour les jeunes gens, son acquisition constituait à peu près un rite de passage dans la tribu technicienne, sans compter que l'objet est esthétiquement agréable à voir, à toucher et à manipuler. Certains en parlent d'ailleurs avec une affectivité non dissimulée, affectivité que suscitent souvent les instruments pour leurs utilisateurs, surtout lorsqu'ils sont emblématiques: «Engineers ever since have carried slide rules at least as lovingly as any physician has ever carried his stethoscope and tongue depressor» (Asimov 1972: 100). Pour un peu, la règle à calcul aurait succédé au compas et à la règle et à l'équerre, puissants symboles anciens de la «raison», un seul exemple entre mille étant le Newton hostilement et superbement dessiné par William Blake. En même temps, pour l'essentiel, son emploi est simple, étant accessible même à qui ignore tout des progressions arithmétique et géométrique et de la fonction logarithmique; voir d'ailleurs dans la bibliographie le titre très aguicheur du deuxième manuel français, anonyme, et datant de 1825 .

Pour une monographie exploratoire, la règle à calcul offre aussi l'avantage de résister à la multiplication des points de vue, qui est source de complexité pour le descripteur. Car, comme le dit Pottier (1992a: 79-80): «Prenons le cas de pont. Dans une statistique économique qui relèvera le nombre de ponts dans une région, on aura le cas limite de l'objet lui-même, hors fonctionnement. Mais en général, chaque objet est lié à un certain nombre de fonctions privilégiées dans un milieu socio-culturel déterminé. Ainsi l'ingénieur des Ponts et Chaussées verra le pont comme l'objet d'une activité constructrice (ACCUSATIF conceptuel), alors que le touriste y verra un lieu de passage (LOCATIF conceptuel) [...]. Plusieurs de ces valeurs sont attachées à une même lexie. Ce qui n'est que combinatoire en français possède une marque morphologique en chinois. La lexie «pont» qiáo prend le spécificatif zuò s'il s'agit de l'ouvrage d'art, et tiào s'il s'agit de la voie de communication». Comme objet technique spécialisé, la règle à calcul est moins sujette à ces modulations de point de vue — si intéressantes, mais si compliquantes - même si elles existent tout de même.

Voici donc quelques suppléments inspirés par la règle à calcul, qui montreront ce qu'enseigne la prise en compte d'un nombre plus élevé de langues. On peut considérer cette petite monographie comme un hommage posthume à un instrument qui a rendu tant de services pendant trois siècles et qui symbolise l'âge classique des instruments mathé- 
matiques, avant de disparaître définitivement et en peu d'années devant les calculatrices électroniques portables, comme, avant lui, avait disparu le compas de proportion. Au moins dans nos contrées, l'objet est devenu en effet une quasi-antiquité, de sorte qu'en 1991, un magasin fort connu de Paris, «La Règle à Calcul», a changé de raison sociale, ne vendant plus depuis longtemps que des ordinateurs, et qu'en août 1992, j'ai pu en voir avec émotion un exemplaire pieusement proposé à des amateurs méditatifs par un brocanteur à La Souterraine, département de la Creuse, au milieu des rogatons les plus hétéroclites: il avait acquis le noble et nostalgique statut de la crémaillère rouillée et du phonographe à pavillon.

Je procéderai par étapes, en rapportant mes données dans l'ordre où elles ont été effectivement collectées, avec les commentaires successifs; l'intérêt de distinguer ces deux phases apparaîtra, même'si, ici ou là, j'ai dû anticiper sur la suite. Il ne m'a pas été possible de rassembler des données dans autant de langues que je l'aurais souhaité (manquent par exemple l'albanais, le gallois, l'afrikaans et les éventuels termes en mongol, tagalog, swahili), parce que les dictionnaires — même assez volumineux — sont souvent muets sur la dénomination de la règle à calcul. On notera d'ailleurs qu'en général, cette lexie ne fait pas l'objet d'une entrée propre, ce qui est significatif du traitement lexicographique en général. Pour d'autres langues (comme le tamoul), l'obstacle de l'écriture m'a arrêté. Dans l'ensemble, cependant, la plus grande part des langues majeures de la technique est représentée. Là où des données m'ont obligeamment été communiquées par des informateurs, leur nom sera cité.

Dans les langues où cela a été possible, les variantes ont été enregistrées (y compris les termes disparus) et notées par des indices. L'emploi des majuscules signalera qu'on est dans le plan sémantique - en notation sémiquement très grossière mais adéquate à nos besoins (voir à la fin pour une comparaison avec l'analyse sémique classique). Ainsi, il va de soi qu'un élément sémantique comme CALCULATEUR serait à décomposer en INSTRUMENT À CALCULER. Mais pour nos besoins, et compte tenu de la procédure, nous ne ferons pas une analyse componentielle suivant les procédures classiques; il s'agira d'une analyse informelle des dénominations, mais qui ne sera pas sans enseignements. Les dénominations théoriquement imaginables mais non attestées sont marquées d'un astérisque. Le signe + désigne une dénomination sortie de l'usage. On n'a pas cherché à unifier strictement les translittérations provenant de diverses sources, ces spécifications étant hors de propos.

J'attire d'ores et déjà l'attention sur certains des concepts un peu vagues de linguistique ou de psycholinguistique molle auxquels j'aurai recours, comme: «schéma définitionnel», «şchéma définitionnel panlinguistique», «représentation sémantique», «saillance», «orientation thématique», «cadre interprétatif», «schéma d'inférence», «méta-schéma d'inférence», «module inférentiel», «filtre lexicogénétique formel». Leur originalité est nulle ou ténue, mais leur utilité éventuelle, ne serait-ce que dans l'élaboration d'un jouet conceptuel, est soumise à l'appréciation du lecteur. Comme on le constatera, je n'entrevois que très obscurément les implications psycholinguistiques d'une telle monographie, même si je me dois de rappeler que, pour Saussure, la linguistique est une branche de la sémiologie, laquelle est à son tour une branche de la psychologie sociale.

\section{B. DESCRIPTION ET DÉFINITIONS}

Il est bon comme apéritif de rappeler assez précisément comment est constituée la règle à calcul. La confrontation de diverses définitions et descriptions n'est pas sans 
utilité. Pour ne pas alourdir le corps de l'article, j'ai rassemblé un assez grand nombre de définitions dans l'appendice $I$, auquel on pourra se reporter.

Comme toujours, la confrontation de ces définitions montre la variabilité et la différence de qualité lexicographiques / encyclopédiques. Je ne veux pas élaborer toute une critique lexicographique, me bornant à quelques observations. Beaucoup de définitions insistent sur la rapidité des calculs, mais il n'est pas fréquemment indiqué que les résultats obtenus sont, dans les cas défavorables, très approchés, ce qui est pourtant important à savoir, puisque cela distingue la règle à calcul d'autres calculateurs. Dans certaines définitions, la morphologie de l'objet, même schématique, est inintelligible, comme avec la règle à coulisse de Littré. On voit à nouveau l'utilité de l'illustration, même très schématique, surtout en technologie (Boisson 1991; De Virville et alii 1977). En comparant Parker et Dorian, on voit que l'un est excessivement abstrait, tandis que l'autre ne néglige pas de préciser qu'il s'agit d'un instrument mécanique, mais même cela reste insuffisant. Dans bien des définitions, on ne comprend pas quels sont les rapports mécaniques précis entre règle, réglette (coulisse) et curseur ni les manipulations auxquelles on soumet ces éléments. D'ailleurs, la plupart des définitions négligent le modus operandi, la séquence opératoire de l'utilisateur, un peu comme une définition de machine à écrire qui ne ferait aucune allusion à la frappe des touches d'un clavier ou une définition de livre qui ne ferait aucune allusion au fait de la lecture par tournement des pages. C'est une carence classique et significative, par quoi l'objet est étrangement envisagé en lui-même, et non dans ses rapports avec l'homme, comme composante d'un système homme-machine à opérations déterminées (cf. Pottier cité plus haut sur le pont). Leibniz, fasciné par les problèmes de description et de recherche documentaire (son ars reminiscendi / revocandi) avait pourtant vu la nécessité de la caractérisation du mode d'emploi, lorsque, dans une lettre à Huyghens sur sa «caractéristique», il disait: «On pourrait faire en caracteres, qui ne seront que des lettres de l'Alphabet, la description d'une machine quelque composée qu'elle pourroit estre, ce qui donneroit moyen à l'esprit de la connoistre distinctement et facilement avec toutes les pieces et même avec leur usage et mouvement sans se servir de figures ni de modelles et sans gener l'imagination, et on ne laisseroit pas d'en avoir la figure présente dans l'esprit autant que l'on voudroit faire l'interpretation des caracteres. On pourroit faire aussi par ce moyen des descriptions exactes des choses naturelles, comme par ex. des plantes et de la structure des animaux» (cité in Dascal 1978: 215216).

Nous voyons aussi à quel point la distinction entre entrée encyclopédique et entrée de dictionnaire est artificielle (Le Ny 1979: 204; Haiman 1980; Boisson, Kirtchuk et Béjoint 1991). Au reste, pour de tels référés (= référents), ne nous faisons aucune illusion de linguiste, l'expérience enseigne que les meilleures définitions sont typiquement celles, développées, que l'on trouve dans les encyclopédies, et non pas dans les dictionnaires, tristement secs et déficients dans ces domaines, et cela, non pas seulement pour des raisons d'espace, mais aussi souvent par désir académiquement puritain d'opposer l'«encyclopédique» au «linguistique». L'interpénétration est pourtant inévitable, comme on 1'a perçu depuis longtemps: «De plus, en expliquant les mots, on est obligé de faire une course dans les sciences mêmes, comme il paraît par les dictionnaires; et de l'autre côté on ne saurait traiter la science sans donner en même temps les définitions des termes» (Leibniz 1765, 1990: livre IV, chap. XXI, 412).

\section{HISTOIRE DE LOBJET RÈGLE À CALCUL}

On ne peut étudier la dénomination d'un objet technique sans étudier l'objet luimême et son histoire, faute de quoi toute monographie terminologique reste «en l'air», et 
la raison de certaines dénominations demeure obscure. Mes sources sont divers ouvrages, articles et encyclopédies cités en bibliographie; je n'ai pu consulter la monographie classique de Cajori, mais on peut supputer que Baxandall en reprend la substance. J'ai cherché à clarifier au maximum la succession des étapes, le plus souvent rendue confuse par des traitements lacunaires, et embrouillee encore par les transmissions fautives d'encyclopédie en encyclopédie.

La règle à calcul est un instrument de choix comme dans une étude de cas technologique, parce qu'il constitue une utilisation simple d'un objet mathématique simple. Le principe en est donc immédiatement disccrnable. La règle à calcul apparaît dans un contexte où les progrès des sciences comme l'astronomie, mais aussi de l'arpentage, de la navigation, de l'artillerie, rendent utiles des améliorations dans les techniques de calcul. Les logarithmes ont été inventés par John Napier (1550-1617), la publication tardive étant de 1614 (Mirifici Logarithmorum Canonis Descriptio ) - et aussi, indépendamment, par Joost Bürgi (publication de 1620), dans un climat intellectuel où l'idée de mettre en correspondance une progression arithmétique et une progression géométrique était dans l'air, ainsi que les recherches de prosthaphairesis, la méthode pour convertir les multiplications en des additions grâce à des formules trigonométriques (sur les travaux de Michael Stifel et de Joost Bürgi, voir: Novy̆ 1970; Ohlmann 1990: 698; Vogel 1976; sur Henry Briggs, voir Huxley 1970). Les travaux de Napier ont été rapidement diffusés: ainsi dès 1620 était publiée à Lyon une traduction de son ouvrage sous le nom de Description et construction des logarithmes (Michaud, article sur Wingate).

La règle à calcul a été inventée au XVII siècle en Angleterre, très peu de temps après l'introduction des logarithmes, et comme généralement en matière d'inventions, en plusieurs stades (comparer par exemple à l'histoire typique, mais évidemment plus complexe, de la bicyclette), trois stades fondamentalement, comme nous le verrons plus loin. La diffusion en a été rapide en Angleterre, considérablement plus lente sur le continent européen.

Il arrive que l'on fasse rentrer dans la préhistoire de la règle à calcul les bâtons de Neper, en anglais Napier's bones ou parfois Napier's rods (Messadié 1988: 168). Si on entend cela précisément, l'assertion me semble très discutable. Napier avait effectivement inventé une «machine» mathématique permettant de ramener la multiplication à l'addition, et la division à la soustraction, système qui se répandit rapidement en Europe et fut largement utilisé au XVII ${ }^{\mathrm{e}}$ siècle, et connut même le succès en Chine et au Japon (Needham et Ling 1959: 72). Cet instrument de calcul est malaisé à décrire sans l'aide d'un dessin: voir les illustrations dans Everyman's Encylopaedia, dans Ligonnière 1987, dans l'article de Baxandall de l'Encyclopeedia Britannica, 14 édition, et aussi dans Baxandall 1926, Flegg 1983, Marguin et alii 1990, Michel 1973, Wynter et Turner 1975, etc. Disons qu'il s'agit d'une collection de bâtonnets indépendants, chacun portant dans des cases gravées tout au long l'ensemble des multiples d'un nombre donné de 1 à 9 . En choisissant les bâtonnets voulus et en les disposant côte à côte sur un support, puis en effectuant certaines opérations de calcul à partir de ce que l'on lit sur les bâtonnets, la «rabdologie» (du grec pour bâton, baguette) permet d'effectuer ingénieusement des multiplications. Les opérations consistent à multiplier suivant la méthode de la gelosia, méthode d'origine arabe ou indienne, déjà bien connue en Europe, spécialement en Italie (Flegg 1983). Messadié commet l'erreur de croire que ce dispositif est une «table à logarithmes mobile». Il n'en est rien, d'abord parce que les compartiments des bâtons ne comportent pas des logarithmes, mais, comme je l'ai dit, les multiples d'un nombre. En deuxième lieu, les bâtonnets, s'ils sont bien disposés en contact les uns avec les autres, ne coulissent pas les uns par rapport aux autres; et d'ailleurs, même si leur simple juxtaposition pouvait suggérer l'idée d'un coulissement possible, il resterait que la relation directe 
avec la règle à calcul serait improbable, car nous allons voir que justement le prototype de la règle à calcul ne comporte aucun coulissement. En fait, un ensemble de bâtons de Neper constitue par assemblage (les bâtons étant des «colonnes») ce que l'on pourrait considérer comme un très approximatif analogue physique d'une «table» numérique, mais répétons que cette table n'a rien de logarithmique. Il est instructif de constater que Napier n'a pas conçu de calculateur fondé sur son invention des logarithmes, mais bien un tout autre genre de calculateur.

Il me semble cependant nécessaire d'attirer l'attention sur l'instrument de calcul le plus important au XVII ${ }^{e}$ siècle (mis à part les jetons, dont l'emploi est bien décrit dans le livre de Flegg, et les abaques), à savoir le compas de proportion (anglais sector, attesté en 1598 chez Thomas Hood, créateur de ce terme) (Daumas 1953; Demoriane 1974: 46; Michel 1973: 22; Singer et alii 1957: 627-628). Demoriane dit justement qu' «en dépit de son nom [il] appartient davantage à la famille des règles et des équerres qu'à celle des compas». Il s'agit en effet d'un compas comportant sur ses branches des échelles graduées (six dans la version aboutie et classique), permettant toute une série de calculs fondée sur les propriétés des triangles semblables, permettant de résoudre de nombreux problèmes arithmétiques et géométriques et remplaçant une bonne dizaine de tables numériques. Ce compas de proportion était en usage notamment chez les artilleurs au début du XVI siècle, mais Galilée semble l'avoir perfectionné (La operazione del compasso geometrico e militare, 1606), se querellant avec Balthasar Capra pour l'attribution d'une invention en fait antérieure à eux. Il était utilisé avec une paire de compas à pointes sèches (et non gradués, ce qui s'appelle dividers en anglais), l'ensemble constituant ce qu'on pourrait appeler un «complexe instrumental» comportant un instrument principal et un instrument ancillaire, comme, par exemple, on a un complexe instrumental pour sculpteur maillet + ciseau. Comme indiqué dans Singer :

The sector remained very popular for calculations in gunnery, surveying, dialling, and gauging, in spite of competition from the slide-rule after that device had been invented about the middle of the seventeenth century. The logarithmic slide-rule did not completely displace the sector until late in the eighteenth century; indeed the sector was included as a standard instrument in drawing-sets and in navigation equipment until well into the nineteenth century.

Des traités détaillés permettaient de s'instruire dans l'usage du compas de proportion, comme, pour se borner à deux exemples français, ceux de Denis Henrion ( $L$ 'Usage du compas de proportion, 1631) ou de Jacques d'Ozanam ( $L$ 'Usage du compas de proportion, 1688). Encore à la fin du XVIII ${ }^{\mathrm{e}}$ siècle, l'Encyclopédie de Diderot et d'Alembert consacre de longues pages à son emploi (tome 3, pp. 751-756).

Nous comprenons mieux maintenant dans quel contexte a été inventée la règle à calcul. Pour démêler l'histoire de son invention, il faudra évidemment distinguer concepteurs, fabricants et descripteurs, et surtout observer que l'élaboration s'est déroulée en trois stades essentiels, plus un stade de perfectionnement non essentiel, de sorte que lorsqu'il faudra se garder de la confusion, je parlerai de «règle de stade $1 / 2 / 3 / 4$ ».

Premier stade : le mathématicien Edmund Gunter (1581-1626), ami de Napier, avait mis au point en 1620 , donc seulement six ans après la publication de Napier, l'ancêtre de la règle à calcul (sur Gunter, voir Pepper 1972). Cette règle de stade 1 consistait en une règle unique de deux pieds graduée logarithmiquement, où l'on effectuait les sommes et soustractions de logarithmes grâce à un compas, qui constitue en quelque sorte une partie nécessaire de ce que l'on pourrait appeler le complexe instrumental de Gunter, de la même manière, observons-le, que le compas de proportion faisait partie d'un complexe instrumental, ainsi qu'on vient de le voir. On peut donc se demander si Gunter n'a pas 
trouvé une partie de son inspiration dans le modèle du compas de proportion (plutôt que des bâtons de Neper), et cela d'autant plus que Gunter est aussi l'auteur d'un compas de proportion, dont Pepper dit: «His account of his sector, in the De sectore et radio of 1623 had circulated in manuscript for sixteen years before its publication. The sector, a development from Hood's, included sine, tangent, logarithm and meridional part scales [...]. With improvements, the British navy used it for two centuries, and it was also a precursor of the slide rule».

Pour être précis, le processus de développement de la règle de stade 1 se serait fait en deux temps: il y a eu d'abord une simple règle logarithmique appelée line of numbers, puis Gunter y a ajouté d'autres échelles pour des usages spécialisés, ce qui a donné le Gunter's scale (Baxandall 1926: 36). Le nouvel instrument obtenu, notre règle de stade 1, est connu sous le nom de Gunter's line (première attestation selon l'Oxford English Dictionary: 1688, mais cela pourrait être antérieur) ou encore Gunter's scale. Gunter luimême, avait joliment appelé son invention logocanon (kanôn = règle en grec), terme non répertorié dans l'OED. Ce terme est examiné plus loin. En français, on parle maintenant de l'échelle de Gunter, ou règle logarithmique, et on trouve aussi règle de Gunter et, sur le modèle anglais, ligne de Gunter (dans d'Ocagne, ibid.). Nous reviendrons plus loin en détail sur les diverses dénominations. La règle de Gunter a été immédiatement connue en France, comme nous le verrons plus loin, tandis qu'en Allemagne, Scheffelt en construisait à Ulm dès 1699 (d'Ocagne, ibid.). Cependant l'écho suscité en France par l'instrument de Gunter est très faible, au point que Joseph Sauveur doit réintroduire une «règle logarithmique» perfectionnée par lui (article sur Gunter dans Michaud; Turner 1988: 258).

Messadié indique que Gunter a «perfectionné l'invention de Napier, bloquant les "osselets" sur une surface»; Flegg (1983: 161-163) établit la même filiation entre les bâtons de Neper et la règle à calcul. Je doute que l'on puisse décrire ainsi l'échelle logarithmique de Gunter, et, comme je l'ai dit, je suis sceptique quant à la filiation des deux inventions, même si dans les deux cas il s'agit d'instruments à calculer, et spécifiquement à multiplier et à diviser. Et en fait, la postérité des bâtons de Neper n'est pas à chercher dans la règle à calcul, puisque, en 1666, Sir Samuel Morland a mis au point un instrument dans lequel les bâtons ont été remplacés par des disques susceptibles de rotation; un instrument analogue, censé avoir appartenu à Napier, est conservé au Science Museum de Londres.

Dans un deuxième stade apparaît la version primitive de la règle à calcul, la règle de stade 2, due au prêtre et mathématicien William Oughtred (1575-1660), connu aussi comme l'introducteur du signe $\times$ pour la multiplication (sur Oughtred, voir Scott 1974). Celui-ci décrit la règle à calcul circulaire dans son traité de navigation manuscrit écrit en latin, traduit en anglais par William Forster sous le nom de Circles of Proportion and the Horizontal Instrument, Both invented, and the uses of both written in Latine by that Learned Mathematician Mr.W. O., et publié en 1632-1633. Une description de ce même instrument circulaire (sous la forme plane et sous la forme cylindrique) avait été publiée précédemment par un de ses élèves, Richard Delamain (dans Grammelogie, or the Mathematical Ring publié en «1630», soit probablement janvier 1631 nouveau style: Turner 1973: 58, note 21), d'où une querelle de priorité entre les deux hommes. On trouvera une photo de cet instrument circulaire dans Turner (1973: planche II, p. 57). Oughtred affirme en tout cas qu'il aurait conçu dès 1621 un instrument rectiligne basé sur l'idée de faire glisser relativement deux règles logarithmiquement graduées et non solidaires, donc maintenues en contact avec une main. Avec Oughtred, le progrès est net, car on élimine l'instrument ancillaire du compas, on passe donc du complexe instrumental à l'instrument unique, mais sous une forme intermédiaire, puisqu'il est composé de deux 
parties non encore solidaires. En outre, la manipulation est ergonomiquement pénible par la crispation qu'elle devait causer, sauf à le poser sur une table, ce qui n'est guère commode non plus.

Dans un troisième stade, les premiers exemples de règles à calcul au sens moderne, les règles de stade 3 , datent de la seconde moitié du XVII ${ }^{-}$siècle, l'innovation consistant à établir une solidarité entre les deux règles (une règle coulisse dans une autre), la prise en main devenant aisée, puisqu'on n'a plus à maintenir deux règles en contact manuellement, comme dans l'invention d'Oughtred. Arrivé à ce stade, l'essentiel de ce qui constitue la règle à calcul moderne est inventé. Le problème est toutefois de déterminer précisément qui est l'inventeur. Le premier exemple matériel de règle attestée date de 1654, il s'agit d'une règle portant 1'indication «Made by Robert Bissaker for $T$. W., l654», et conservée au Science Museum de Londres (description dans Baxandall 1926: 39); Bissaker est donc le fabricant, et non pas le concepteur. En 1657, Seth Partridge fait connaître la règle à calcul en écrivant son manuel d'utilisation de l'instrument, publié quatre ans plus tard (The Description and Use of an Instrument Called the Double Scale of Proportion, Londres 1661), de sorte qu'il est souvent considéré comme l'inventeur de la règle de stade 3. Sur Seth Partridge, qui se présentait lui-même comme «Surveyor and Practitioner in the Mathematicks», et qui est également l'auteur d'un livre sur les bâtons de Neper, Rabdologia, or the Art of numbering by Rods, 1648, on ne sait pas grand-chose (voir l'article dans le Dictionary of National Biography). Anecdote peut-être significative, l'introduction dans un pays aussi lointain que la Chine a été remarquablement rapide, comme le montre une vénérable règle chinoise datable de 1660 ou après (dans Needham et Wang Ling 1959, figure 68, en face de la page 74, photo reproduite d'un article de H. Michel), instrument d'allure très «moderne», avec la règle et la réglette coulissante.

Noter en passant que le pied à coulisse, dont nous reparlerons brièvement plus bas, aurait pu servir de modèle et suggérer une adaptation-transfert assez directe pour une conception directe de la règle à calcul de type 3, mais que cela n'a pas été le cas, si bien que cette dernière a été inventée par tâtonnements progressifs le long d'une trajectoire autonome d'innovations.

On attribue aussi fréquemment au mathématicien Edmund Wingate (1593-1656) l'idée de coulissement relatif de deux règles, mais il s'agit là d'une confusion, car Wingate est seulement responsable de l'introduction de la règle de Gunter en France dans son livre L'Usage de la Reigle de Proportion en l'Arithmétique \& Géométrie, Paris 1624, ouvrage ensuite traduit en anglais sous le titre de The Use of the Rule of Proportion, qui a connu cinq éditions de 1626 à 1683 (sur Wingate : articles dans le Dictionary of National Biography, dans Michaud). Wingate a aussi publié en 1628 à Londres The Construction and Use of the Line of Proportion. Certaines sources superficiellement informées vont même jusqu'à attribuer par erreur et trop tardivement l'invention de la règle à calcul à coulisseau (règle de stade 3) tantôt au Français Étienne Lenoir, grand constructeur d'instruments de précision et réalisateur du mètre étalon de platine, tantôt à Leadbetter, astronome anglais du XVIII ${ }^{\mathrm{e}}$ siècle (l'encyclopédie Comment ça marche, la Grande enciclopedia portuguesa e brasileira, le $Q$ uid). Si certains ouvrages confondent concepteurs et descripteurs, d'autres ouvrages confondent concepteurs et fabricants (ainsi le dictionnaire de Lami, 1882).

Comme on le voit, l'invention de la règle à calcul circulaire (plane ou cylindrique) suit de peu celle de la règle rectiligne d'Oughtred. De même, une disposition en «spirale» apparaît en 1633 avec Thomas Brown, la règle hélicoïdale apparaîtrait dès 1650 avec Milburne. Donc, une fois établie la règle d'Oughtred, la «famille» des règles à calcul commence à se constituer en une trentaine d'années. La famille ne cessera de s'élargir, mais les détails importent peu pour nous ici. 
La règle à calcul connaît un grand succès en Grande-Bretagne, même si le compas de proportion y demeure en grand usage comme son concurrent; de nombreuses professions, et jusqu'aux jaugeurs, douaniers et commis de l'octroi, en font usage; le manuel français anonyme de 1825 avance même, non sans une probable hyperbole anglomane, que l'instrument «est entre les mains des plus simples ouvriers». Selon Everard, l'un des principaux fabricants anglais du XVII e siècle, plusieurs milliers de règles auraient été vendues entre 1683 et 1705 . Très vite, elle donna lieu à toutes sortes d'adaptations aux besoins de diverses professions: règles nautiques, financières, de jauge, pour les éleveurs de bestiaux, etc. En France, Sauveur essaiera de promouvoir l'instrument vers 1700, mais sans succès. On le voit bien au silence de l'Encyclopédie de Diderot et d'Alembert, très inadéquatement compensé par le volume 3 du supplément paru en 1777, dans un article («Logarithmiques (baguettes, échelles, règles)») qui témoigne d'une ignorance caractéristique de l'invention anglaise et fournit un historique inexact. Il faudra qu'en 1815 l'ingénieur et géographe Edme François Jomard présente la règle (Bulletin de la Société d'encouragement pour l'industrie nationale) telle que la fabriquait Jones à Londres pour qu'elle soit enfin adoptée, et dès lors avec grand succès; Jomard fera d'abord fabriquer la règle par le fils de Lenoir. Les manuels d'utilisation se succèdent alors rapidement à partir de 1820 (Collardeau 1820, à partir d'un modèle anglais, réédition de 1833; Anonyme 1825; Artur 1827). De même, en Allemagne, Lambert d'Augsbourg, qui fit exécuter «des grandes règles de quatre pieds de long» (Artur 1827: I), ne semble pas avoir été suivi. La règle passera en Italie au XIX ${ }^{\mathrm{e}}$ siècle (voir le manuel de Sella, 1859); même chose pour l'Autriche. La résistance à la diffusion de la règle à calcul n'est pas claire, mais cette petite énigme mériterait une investigation. On ne voit pas que le prix de l'objet puisse ici jouer un rôle significatif. Convient-il d'invoquer la différence dans la législation des brevets? Faudrait-il faire intervenir certains partis-pris nationaux? On sait comment, sinon en technique, du moins en science, les Français ont été un temps rétifs à la mécanique de Newton, à la génétique de Morgan, et inversement, les Anglais à la chimie de Lavoisier. Cependant, souvenons-nous que l'adoption des tables de logarithmes a été immédiate en France (l'adoption des innovations mathématiques est-elle plus rapide?). En tout cas, une étude comparée fine et interprétative de la conception et de la diffusion des instruments dans divers pays européens reste à faire.

En 1851, le Français Amédée Mannheim (1831-1906), alors jeune lieutenant d'artillerie, perfectionne la règle en y adjoignant une partie mobile non essentielle mais très utile dans la pratique: le curseur de verre avec un trait gravé, ce qui donne la règle telle que nous la connaiss(i)ons, qu'on pourrait appeler règle de stade 4 (sur Mannheim, qui devint professeur de géométrie à l'École Polytechnique, voir Koppelman 1974). Le curseur a en fait été «réinventé» par Mannheim, l'idée et même la réalisation étant déjà connues très antérieurement, de Newton parmi bien d'autres, comme on le voit dans le manuel anonyme de 1825, pages 116-117; voir aussi Baxandall 1926: 38, 48. Noter au passage que le mot curseur désignait auparavant le nombre 1 pris sur la coulisse (voir Anonyme $1825: 16$, note 1 ). Il est curieux de constater que cette innovation du curseur a avorté de manière répétée avant Mannheim. La technique n'était-elle pas au point? Fautil invoquer des paramètres commerciaux ? En effet, à partir de 1880, la règle de stade 4 (le système Mannheim, la règle Mannheim), fabriquée par la maison parisienne TavernierGravet, héritière d'améliorations substantielles dans la finesse de la graduation, est largement exportée dans de nombreux pays, y compris l'Allemagne et, par une superbe ironie, la Grande-Bretagne. Les innovations postérieures sont plus secondaires, comme la substitution des plastiques à l'ivoire (qui a des incidences sur la lisibilité des graduations autant que sur le prix de l'objet), la gravure de trois traits sur le curseur au lieu d'un seul (faci- 
litant certains calculs), l'adjonction d'une loupe au curseur (autorisant une précision plus grande à longueur égale), etc.

On devra avoir présent à l'esprit qu'avant 1890 , seuls trois pays fabriquaient des règles à calcul et les exportaient: l'Angleterre (maison Davis, de Derby), la France (d'abord Paul-Étienne Lenoir, fils d'Étienne Lenoir, puis le successeur Gravet, puis le successeur Tavernier-Gravet) et l'Allemagne (Nestler, Faber, Keuffel et Esser). Ce fait ne peut manquer d'avoir eu des incidences linguistiques.

\section{DÉNOMINATIONS DANS DIVERSES LANGUES : PREMIÈRE COLLECTE DE DONNÉES}

1) ANGLAIS

slide-rule ou slide rule (première attestation dans Pepys, qui avait acheté un instrument à John Brown, 1663), et aussi sliding rule, qui semble plus rare (première attestation également dans Pepys, 1663: c'est l'entrée dans le Imperial Dictionary de 1851); enfin la variante †sliding scale (1706), totalement inusitée maintenant, même si elle est encore donnée dans le dictionnaire de Kettridge.

\section{2) FRANÇAIS}

règle à calcul, règle à calculer, trègle logarithmique (ce dernier terme semble maintenant inusité; on le trouve comme variante dans Artur 1827: I); le Grand Larousse de la Langue Française fait remonter la première attestation de règle à calcul à Bescherelle (1845), mais en fait règle à calcul figure déjà dans un manuel anonyme de 1825 (voir plus loin).

3) ITALIEN

regolo calcolatore.

\section{4) ALLEMAND}

Rechenschieber.

Formé sur Rechnung, calcul, rechnen, calculer, et sur Schieber, coulisseau, curseur, etc. Coulisseau est ainsi défini dans le Quillet: «Toute pièce mâle qui se meut dans une coulisse partie femelle. Ex. : coulisseau d'une règle à calcul».

\section{5) RUSSE}

logarifmíceskaja linéjka [= règle logarithmique] (les accents aigus notent l'accent dans la translittération, pour aider à la prononciation).

6) TCHEQUE

logaritmiké pravítko [= règle logarithmique]; počítací pravitko [règle à calculer] (les accents aigus notent la longueur vocalique dans l'orthographe tchèque).

\section{7) HÉBREU}

sargel hil $u v$

Formé par assemblage à l'état construit sur sargel, règle, et hiluv, calcul (information de Pablo Kirtchuk).

\section{E. COMMENTAIRES SUR LA PREMIÈrE COLLECTE DE DONNÉES}

La confrontation de ces diverses langues permet de bien voir comment chacune sélectionne des caractéristiques de l'instrument, puisque les dénominations sont glosables de la manière qui va suivre. Comme nous serons sur le plan sémantique, nous ne nous 
intéresserons pas à ce qui, pour notre objet, est le résultat de contraintes formelles propres à chaque langue, comme le fait qu'une langue ait règle à calcul ou règle à calculs, ou règle de calcul, ou règle à calculer, ou règle calculante, ou règle calculatrice: ce ne seront là que des variantes de réalisation d'une même représentation sémantique. Nous regrouperons donc les termes attestés en ces quatre représentations sémantiques très informelles :

— RÈGLE À CALCUL(ER) (français, ${ }_{1}$, italien, hébreu, tchèque ${ }_{2}$ )

- RÈGLE GLISSANTE / À COULISSEAU (anglais)

- COULISSEAU À CALCUL (allemand)

— RËGLE LOGARITHMIQUE (français ${ }_{2}$, russe, tchèque ${ }_{1}$ )

Certains éléments des dénominations sont morphologiques, descriptifs (en incluant dans ces termes, par léger abus de langage, ce qui réfère au mode d'utilisation, comme l'idée de faire coulisser une partie par rapport à une autre), d'autres fonctionnels (à quoi sert l'objet). Mais si l'on veut être rigoureux, il faut distinguer ces quatre ensembles de caractérisations : 1) comment est constitué l'objet (morphologie) ; 2) comment on l'utilise (manipulation); 3) à quoi il sert (fonction); 4) sur quelles bases intellectuelles il a été conçu pour remplir sa fonction (principe). haut.

Parlante est la confrontation avec les définitions des dictionnaires données plus

On voit que le strict minimum pour avoir un noyau définitionnel (encyclopédiquement insuffisant, bien sûr) serait quelque chose comme le schéma définitionnel suivant: (1) RĖGLE(S) (2) COULISSANTE(S) (3) POUR CALCULER (4) AU MOYEN DE LOGARITHMES. On pourra considérer que la notion de RĖGLE inclut la notion de GRADUATION. Maintenant, si l'on essaie de fabriquer a priori des représentations sémantiques binaires à partir du schéma définitionnel en question, on voit que 6 schémas sont envisageables, glosables comme suit:

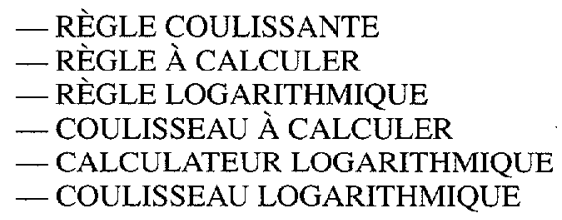

Au surplus, l'observation montre que malgré l'existence d'une «règle» et d'une «réglette», aucune langue jusqu'ici ne parle de «règles» au pluriel, et on peut s'attendre à ce que de telles dénominations soient rares.

J'ai simplifié un peu, parce que je m'intéresse seulement aux combinaisons de sèmes deux à deux. Sinon je devrais distinguer l'ordre des sèmes dans une linéarisation mimant ce que, par un léger abus de langage, on pourrait appeler l'orientation «thématique» de la représentation sémantique, suivant ce qui est sélectionné comme thème (anglais topic) ou comme rhème (anglais comment), le terme représentant en somme une proposition ramassée, comme je l'avais indiqué dès le début. On pourrait donc opposer COULISSEAU À (= POUR) CALCULER s'opposant par renversement à CALCULATEUR À (= AVEC) COULISSEAU / À GLISSEMENT. Formellement, cela se réalise par un syntagme avec un modifié (une «tête») et un modificateur. A priori, on pourrait imaginer que des langues puissent jouer sur cette différence dans la structure thématique, qui, d'une manière générale, devrait être soigneusement étudiée en terminologie, puisque le membre thématique déclenche la catégorisation première. Cette possibilité exprimée ici 
linguistiquement comme le renversement dans l'orientation thématique est anciennement connue sous une forme logique:

Au reste il est encore bon de remarquer que bien souvent le genre pourra être changé en différence, et la différence en genre, par exemple: le carré est un régulier quadrilatéral, ou bien un quadrilatère régulier, de sorte qu'il semble que le genre ou la différence ne diffèrent que comme le substantif et l'adjectif; comme si au lieu de dire que l'homme est un animal raisonnable, la langue permettait de dire que l'homme est un rational animable, c'est-à-dire une substance raisonnable douée d'une nature animale; au lieu que les génies sont des substances raisonnables dont la nature n'est point animale, ou commune avec les bêtes. Et cet échange des genres et différences dépend de la variation de l'ordre des sous-divisions. (Leibniz 1765, 1990: livre III, chap. III, 226-227)

On peut encore raffiner si l'on introduit explicitement la notion d'échelle (logarithmique), qui se trouve d'ailleurs bel et bien dans le terme anglais obsolète $\nmid$ sliding scale (où scale est métonymique pour «rule on which scales are engraved»; maintenant sliding scale désigne uniquement l'«échelle mobile» au sens économique). Cette notion n'est en effet qu'implicite dans la notion englobante de RĖGLE, puisqu'une règle est un objet rectiligne (une planchette) non nécessairement gradué. Bien entendu, une règle graduée sert à mesurer, tandis que les règles d'une sliding rule servent à calculer (mais voir plus loin un terme anglais ancien qui entretient la confusion).

Il faudrait alors partir de la définition approximative que voici (schéma définitionnel $n^{\circ}$ 2): (1) RËGLE(S) (2) COULISSANTE(S) (3) POUR CALCULER (4) AU MOYEN D'ÉCHELLES (5) LOGARITHMIQUES. Ou encore: (1) PLANCHETTE(S) (2) GRADUÉE(S) (3) EN ÉCHELLES LOGARITHMIQUES (4) POUR CALCULER. Ceci ferait attendre a priori 10 représentations sémantiques (binaires).

Le terme de Gunter, logocanon, peut s'interpréter de manière ambiguë, à cause du reste d'une ambiguïté originelle sur le terme logarithme lui-même (dû à Napier, 1614, sous la forme latine logarithmus), dont l'OED dit ceci : «Napier does not explain his view of the literal meaning of logarithmus. It is commonly taken to mean "ratio-number", and as thus interpreted it is not inappropriate, though its fitness is not obvious without explanation. Perhaps, however, Napier may have used "logos" merely in the sense of "reckoning", "calculation" [cf. LOGISTIC]». Faut-il interpréter le log- du logocanon de Gunter comme une abréviation de logarithmus (auquel cas logocanon signifierait RĖGLE LOGARITHMIQUE), ou bien comme le deuxième sens donné dans la citation de l'OED à propos de logarithmus (auquel cas logocanon signifierait RÈGLE À CALCUL(ER)) ? I1 est vraisemblable cependant que la première interprétation de l'OED est la bonne, la notion de «rapport», de «proportion» étant ici capitale (voir plus loin). Quoi qu'il en soit, le terme logocanon n'a eu aucun succès, malgré sa commode compacité, et le terme usité était Gunter's line / scale.

Lorsque l'innovation du coulissement relatif de deux règles a été imaginée, la solution immédiate pour la dénomination a été effectivement de nommer ce nouvel objet par rapport au précédent - et c'est pourquoi, en terminologie, l'étude des dénominations ne doit pas négliger l'aspect historique pour les objets (étude technologique de l'objet), diachronique pour les termes (étude linguistique du mot). C'est ce qui, me semble-t-il, justifie les termes de slide-rule et sliding rule. On aurait pu penser en effet que la première dénomination de la règle à calcul aurait pu être quelque chose comme règle logarithmique (comme on fait en russe et en tchèque). Mais justement la règle de Gunter se trouvait déjà être une règle logarithmique, et ce qui différencie la règle à calcul dûment constituée par rapport à la règle de Gunter, c'est l'innovation du coulissement, 
d'où le logiquement attendu slide-rule. Nous serons conduits à revenir là-dessus et à examiner plus loin des termes anglais maintenant disparus.

Comme les Anglais ont été les innovateurs de ces instruments, on aurait pu penser que les autres langues allaient se contenter de calquer leurs dénominations sur l'anglais, qui fournit, notons-le, un terme purement morphologique, descriptif. Mais on voit qu'il n'en a rien été. L'allemand a bien gardé l'idée de coulissement, mais a choisi une dénomination mixte morpho-fonctionnelle (COULISSEAU À CALCUL). Le français et l'italien ont aussi une dénomination mixte morpho-fonctionnelle (RÈGLE À CALCULER), ainsi que le russe et le tchèque (RËGLE LOGARITHMIQUE), encore qu'ici la description fasse appel à une entité abstraite (le logarithme) dont la règle porte des traces matérielles sous la forme d'échelles graduées.

La question se pose immédiatement: existe-t-il des langues dans lesquelles on trouverait les deux dénominations manquantes dans la liste des six types dérivables des représentations sémantiques fabriqués a priori ci-dessus (sans parler de langues éventuelles où le composé serait ternaire, hypothèse économiquement peu probable)?

Notre schéma définitionnel laisse attendre le remplissage des deux lacunes dénominationnelles par des langues figurant dans un échantillon plus développé. II possède donc un pouvoir prédictif qui sera à confronter avec des données supplémentaires.

$\mathrm{Si}$ on s'apercevait que les trous sont systématiques (soit absolument, parce que les deux lacunes dénominationnelles ne sont remplies par aucune langue, soit relativement, parce que peu de langues les remplissent), cela pourrait-il s'interpréter comme un fait aléatoire, ou comme fournissant des indications sur certaines préférences, certaines règles, certaines contraintes dans la dénomination? Cela mériterait d'être étudié systématiquement sur un ensemble d'objets, car on pourrait alors détecter éventuellement des régularités générales pesant sur les contraintes de dénomination, régularités interprétables interactionnellement d'une part en fonction des caractéristiques des objets, d'autre part des caractéristiques psychologiques des utilisateurs.

Malgré l'infime échantillon de langues jusqu'ici rassemblé, notre modèle laisse aussi attendre quels types de dénominations seront prédominants, à savoir d'une part RÈGLE À CALCUL(ER) (représenté par: français ${ }_{1}$, italien, hébreu, tchèque ${ }_{2}$ ) et RÈGLE LOGARITHMIQUE (représenté par: français ${ }_{2}$, russe, tchèque ${ }_{1}$ ).

\section{F. DÉNOMINATIONS DANS DIVERSES LANGUES : DEUXIÈME COLLECTE DE DONNÉES}

Aux sept langues déjà citées, nous ajouterons maintenant celles-ci, avec quelques ajouts pour certaines des sept langues précédentes. Je n'ai pas hésité à faire figurer l'espéranto, qui, quoique langue artificielle (de structure indo-européenne, mais agglutinante sans allomorphie pour ce qui est du lexique), présente bien des caractères de naturalité intéressants; elle constitue un objet linguistique digne d'étude et est notamment pleine d'enseignements pour la lexicogenèse; il n'y a donc aucune raison de la négliger, comme on le fait par indifférence ou ignorance (le lecteur intéressé par la formation des mots en espéranto pourra consulter: Janton 1977; Kalocsay et Waringhien 1985; Wells 1969; Wells 1989). Je réserverai les ajouts de l'anglais pour une section spéciale suivante, vu l'importance de l'Angleterre dans l'invention de l'instrument.

\section{2) FRANÇ.AIS}

Autres dénominations: règle à calculs (ce pluriel, probablement obsolète, dans $L a$ Grande Encyclopédie, sub arithmomètre, tome 3; déjà dans Artur 1827, dans Collardeau 1833); †règle glissante (Bescherelle, Dictionnaire national, 1850), qui doit être un calque de l'anglais, mais qui ne semble plus employé depuis longtemps; un autre calque 
de l'anglais (voir plus loin) est †règles de proportion (ceci est donné comme étant la première dénomination dans d'Ocagne 1905: 112); se rappeler aussi qu'Henrion avait parlé de règle proportionnelle pour le logocanon de Gunter.

3) ITALIEN

Autre dénomination: regolo per contare.

4) ALLEMAND

Autre dénomination: Rechenstab (= bâton à calculer).

5) RUSSE

Autre dénomination: sčëtnaja linejka (= règle calculatrice), dans la Bol'čaja sovetskaja enciklopedija (1954).

8) ESPAGNOL

regla de cálculo.

9) PORTUGAIS

régua de calculo.

10) CATALAN

regle de càlcul.

11) ROUMAIN

riglă de calcul.

12) BRETON

reolenn-jedi / jediñ (= règle à calculer).

13) IRLANDAIS

rialóir sleamhnáin [ria 'lo: $\mathrm{rj}$ · $\int_{1} \mathrm{j}$ jaunj] (= règle glissante).

14) GALLOIS (information de Paul Meara, Swansea)

llithriwl (sur llithro glisser + riwl règle); aussi riwl rifo (rifo < rhifo calculer).

15) GREC

logarithmikós kanốn (= règle logarithmique).

16) DANOIS

regnestock, sur regne (calculer) et stok (bâton, baguette).

17) NORVÉGIEN

regnestav, sur regne (calculer) et stav (bâton).

18) SUÉDOIS

räknesticka (= bâton à calculer).

19) ISLANDAIS

reiknistrika (= règle à calculer); reiknistokkur (où stokkur désigne le plumier, donc l'idée d'une planchette coulissante) (information d'Emil Eyjolfsson). 


\section{0) NÉERLANDAIS} calculer).

rekenliniaal, sur reken (calculer) et liniaal (règle); rekenschuif (= coulisseau à

\section{1) SLOVAQUE}

logaritmické počitadlo (počitadlo = boulier, compteur).

22) POLONAIS

suwak logarytmiczny (suwak = tiroir, puis, techniquement: glissoir, coulisseau, curseur).

23) SERBO-CROATE

računalno ravnilo (= règle [ravnilo] à calculer).

24) SLOVÈNE

računalo ravnilo (= règle [ravnilo] à calculer).

25) BULGARE

smetačna linija (= règle à calculer).

26) LETTON

logaritmiskais lineāls (= règle logarithmique), vraisemblablement sous l'influence du terme russe (information d'Andrejs Bankavs, Riga).

27) ALBANAIS (information de Christian Gut, EPHE)

Deux expressions avec vizor / je-a ou rig / $\ddot{e}$-a (raie, sillon > règle) + njehsimi ou blogaritjeje (calcul, à l'ablatif).

28) PERSAN (information de Xavier Lelubre, Université Lyon 2)

hatțkě̌e mohāsebe, satâre-ye hesāb (tous deux = règle de calcul).

29) HINDI

slide rule, emprunt direct à l'anglais, qui est pratiquement le seul terme utilisé ; visarp $\bar{l}$ ou sarpi $\bar{l}$, du substantif sanskrit sarpan, action de glisser lentement < sarp, serpent; parikalan-pattika sur parikalan, mesure et pattika, petite bande, petite latte (information de Nicole Balbir, INALCO, Paris).

30) BENGALI Paris).

slide rule, emprunt direct à l'anglais (information de France Battacharya, INALCO,

31) HONGROIS

számololéc, sur számol, calculer et léc, latte, lamelle de bois, planche; logarléc [= planchette logarithmique] (les accents notent les voyelles longues).

32) FINNOIS

$$
\text { laskutikku }(\text { lasku }=\text { calcul }+t i k k u=\text { bâton }) .
$$

33) ARABE (informations de Xavier Lelubre et de Joseph Dichy, Université Lyon 2)

mistara hăsiba (= règle calculante, calculatrice); misțara munzaliqa (= règle coulissante); une autre dénomination dialectale était connue au Liban: mastarat muhandis (= règle d'ingénieur). 
34) TURC

hesap cetveli, sur hesap, calcul (venu de l'arabe) et cetveli, tableau, table, rôle, liste, règle.

35) VIETNAMIEN

th $u^{\supset} \sigma^{\supset} \mathrm{C}$ tính, sur $t h u^{\supset} \sigma^{\circ} \mathrm{C}$, règle et tính, calcul.

36) CHINOIS

chit $^{4}$ suan $^{4} \mathrm{ch}^{\prime}{ }^{3}{ }^{3}$, qui, malgré les apparences, est un composé binaire et non ternaire, puisque $c^{\prime}{ }^{\prime} h^{3}=$ règle et $c^{4} i^{4} \operatorname{suan}^{4}=$ calcul (les deux termes signifient «compter, calculer», et constituent un composé redondant comme les aime le chinois: information de Léon Thomas). Les exposants numériques notent les tons.

37) JAPONAIS

keisanjaku (information de Jean Cholley), sur kei = mesurer, san = compter, et jaku $=$ règle. Le parallèle avec le chinois est évident, bien que mon informateur le glose «règle à comptes et mesures».

\section{8) CORÉEN}

kyesan-cha (information de J. H. Yeon, SOAS, Londres), terme sino-coréen glosable «règle à calculer», à comparer au terme japonais.

39) INDONÉSIEN

mistar hitung (= règle, venu de l'arabe + calcul).

40) BASQUE

kalkulu erregela (information de Michel Morvan).

\section{1) ESPÉRANTO}

glitkalkulilo (= calculateur à glissement, dans Waringhien (Ed.). 1970); kalkulbastono (= bâton à calcul, dans Waringhien (Ed.). 1970, repris dans Wells 1969); logaritma kalkulilo (= calculateur logarithmique, formation de certains mathématiciens espérantistes rapportée par François Lo Jacomo); linia kalkulilo (=calculateur linéaire / à règle, formation personnelle de Lo Jacomo, voir plus bas); pour être complet, citons aussi kalkulmetro (formation avec le suffixe -metro, dans Waringhien (Ed.). 1970, mais c'est plutôt un terme générique qui englobe glitkalulilo).

\section{G. AUTRES DÉNOMINATIONS ANGLAISES : DEUXIÈME COLLECTE DE DONNÉES}

Nous devons être attentifs aux dénominations anglaises anciennes. Je vais donc reprendre l'ensemble des termes que j'ai pu rassembler, sans garantir qu'il n'en existe pas d'autres encore.

Pour commencer par la règle de stade 1, la règle de Gunter, je rappelle les termes déjà mentionnés line of numbers, Gunter's scale, Gunter's line, et le terme logocanon de Gunter lui-même. Un autre terme est employé par Wingate dans son ouvrage publié à Londres en 1626: rule of proportion; le même Wingate emploie line of proportion dans The Construction and Use of the Line of Proportion, Londres, 1628. L'instrument est appelé line of proportion / line of numbers dans William Leybourn, The Line of Proportion or Numbers Commonly Called Gunter's Line Made Easie [...] Whereunto is added, the Use of the Line of Proportion Improv'd, Londres, 1667. John Brown appelle également l'instrument line of proportion / line of numbers dans sa publication londonienne de 1670, A Supplement to the Line of Proportion or Numbers, Containing the 
Description, and Some Uses of a Convenient Two-Foot Joynt Rule. En français, on a d'abord utilisé logocanon et règle de proportion dans l'ouvrage de Wingate publié en 1624 à Paris, ou encore règle proportionnelle dans l'ouvrage de Denis Henrion, Logocanon ou règle proportionnelle, sur laquelle sont appliquées plusieurs lignes et figures, divisées selon diverses proportions \& mesures, en faveur de ceux qui se délectent en la pratique des divines Mathématiques, Paris, 1626 (d'Ocagne 1905: 105 ; Itard 1972; Turner 1988: 255, note 12). Je rappelle aussi pour le français : échelle de Gunter, règle de Gunter, ligne de Gunter, règle logarithmique.

Pour ce qui est de la règle de stade 2, l'instrument primitif d'Oughtred (qui, je le rappelle, comprend deux règles maintenues en contact par la main), on trouve rulers of calculation dans Two rulers of calculation, la première publication (1633) décrivant cet instrument. Il est amusant de constater que c'est un lointain prototype (au pluriel) de la règle à calcul française, qui n'a eu pourtant aucune descendance en Angleterre.

Pour la règle de stade 3 (comme l'instrument construit par Bissaker), on trouve ces termes, dont seuls les deux premiers demeurent:

1) Sliding rule. «The term "sliding rule", which appears to have been first used by John Brown in 1662, was adopted generally by the end of the 17th century» (Baxandall 1926: 38). John Brown était fabricant de règles. Le terme est attesté chez Pepys en 1663.

2) Slide rule (Pepys 1663).

3) †Double scale of proportion, dans le manuel de Seth Partridge, The Description and Use of an Instrument Called the Double Scale of Proportion, Londres, 1661 (livre écrit en 1657). Ce terme est instructif, à cause du terme français compas de proportion; mais ne pas oublier que l'anglais disait et dit normalement sector, bien qu'on rencontre aussi proportional compasses (y compris chez Thomas Young en 1807), ou même proportional divider (ainsi dans une traduction du français chez Itard 1972: 271).

On notera aussi dans l'OED, sub proportional, une citation de 1710 prise dans un dictionnaire technique: «Proportional Scales, sometimes also called Logarithmical; are only the Artificial Numbers or Logarithms placed on Lines, for the ease and avantage of Multiplying, Dividing, Extracting Roots, etc, by means of Compasses or by SlidingRules».

4) $\dagger$ Rulers of proportion, terme employé par l'astronome John Flamsteed en 1673 (cité dans Baxandall 1926: 37).

5) †Gauging-rod, dans Thomas Everard, Stereometry made easie: or, the Description and Use of a new Gauging-Rod or Sliding-Rule, 1684 (cité dans Baxandall 1926: 39, item $\mathrm{n}^{\circ} 99$ ).

6) †Sliding Gunter, terme signalé par Baxandall comme étant utilisé par le fabricant William Robertson (1926: 38).

7) †Sliding scale (1706).

8) †Soho rule. On dit souvent que c'est parce que les règles étaient fabriquées à Soho (d'Ocagne 1905: 115, note 1; Marguin et alii 1990: 39). En fait, on ne peut pas superposer Soho rule à un terme comme Soho tapestry (tapisseries faites en particulier dans le quartier de Soho à Londres après 1685). D'abord, mais c'est un détail, il s'agit non pas du Soho de Londres, mais du Soho près de Birmingham, où se trouvaient les usines de l'entrepreneur et ingénieur Matthew Bolton, devenu associé de James Watt. Ensuite, rien ne permet de dire que les règles étaient fabriquées dans l'usine de Soho. Elles étaient fabriquées à partir de 1779 , avec diverses améliorations dont une plus grande précision dans la graduation, pour les ingénieurs, contremaîtres et superior workmen qui travaillaient sous la direction de Bolton et de Watt dans l'usine de Soho. La Soho rule ne semble donc pas désigner nécessairement toute espèce de règle à calcul, mais un type de règle pour ingénieurs (voir Baxandall 1926: 38). «The habit of using it upon all occa- 
sions is almost confined to those who have been educated at Soho» est-il dit par J. Farey dans son Treatise on the Steam Engine (1872), cite dans Baxandall (1926: 43). On touche ici de très près à la connotation, puisque le terme renvoie à un milieu très restreint de techniciens d'une entreprise bien déterminée, à la pointe de la Révolution industrielle.

9) †Soho scale, variante du précédent (donné dans d'Ocagne 1905, 115, note 1).

On notera qu'au XVII ${ }^{e}$ siècle, la règle à calcul pouvait être informellement classée dans les instruments de mesure, les jauges, les gauging rules («the sliding rule [...] and all sorts of gauging instruments» dit une publicité citée dans Bryden 1992: 321). On notera aussi dans la citation de 1710 que le compas de proportion et la règle à calcul sont cités ensemble.

\section{H. COMMENTAIRES SUR LA DEUXIÈME COLLECTE DE DONNÉES}

Pour la règle de stade 3 , nous sommes maintenant en position de compléter notre liste de possibilités dénominationnelles de la sorte (d'autres représentations sémantiques suivront plus loin cette première liste):

- RËGLE COULISSANTE / À COULISSEAU (anglais ${ }_{1}$, anglais ${ }_{2}$, irlandais, gallois ${ }_{1}$, hindi $_{1}$ [emprunt], bengali [emprunt], arabe ${ }_{2}$, français $_{3}$, islandais implicitement et $_{2}$ indirectement);

- RËGLE À CALCUL(ER) (français, ${ }_{1}$, italien, , italien $_{2}$, espagnol, catalan, portugais, roumain, islandais ${ }_{1}$, breton, gallois ${ }_{2}$, néerlandais ${ }_{1}$, russe $e_{2}$, tchèque ${ }_{2}$, serbo-croate, slovène, bulgare, albanais ${ }_{1}$, albanais al $_{2}$ hébreu, arabe ${ }_{1}$, turc, vietnamien, chinois, japonais, coréen, indonésien, basque);

- REGLE LOGARITHMIQUE ( français $_{2}$, russe $_{1}$, tchèque 1 , letton, grec);

- COULISSEAU À CALCUL(ER) (allemand ${ }_{1}$, néerlandais , $_{2}$ );

- CALCULATEUR LOGARITHMIQUE (slovaque, espéranto s $_{3}$;

- CALCULATEUR LINÉAIRE / À RÈGLE (espéranto ${ }_{4}$ );

- CALCULATEUR COULISSANT / À COULISSEAU (espéranto ${ }_{1}$ );

- COULISSEAU LOGARITHMIQUE (polonais).

Que nous enseigne notre deuxième collecte de données? Nous répartirons nos conclusions en trois rubriques essentielles, en tenant compte de schémas généraux pour la formation des termes (sur certains de ces mécanismes lexicogénétiques en terminologie, voir la mise au point de Quemada 1978).

\section{1) CONFIRMATION OU INFIRMATION DU MODÈLE}

Premièrement, conformément à ce que pouvait laisser attendre le modèle, les deux lacunes terminologiques qui restaient après notre première collecte de données sont maintenant dûment remplies par le slovaque, d'une part (et par une des variantes de l'espéranto), et par le polonais, d'autre part.

Deuxièmement, conformément à ce que nous pouvions attendre, les dénominations les plus fréquentes sont réductibles de façon massivement prédominante au type RÈGLE À CALCUL(ER). Il s'agit d'un sous-type de la dénomination fonctionnelle la plus simple, illustrée par des termes comme machine à écrire, adding machine, washing machine, sewing machine (sous la forme la plus générale, avec le générique machine), ou compte-pas, compte-tours, compte-secondes, compte-fils, compte-gouttes, pèse-lait, pèsegouttes, pèse-acide, pèse-alcool, pèse-moût, pèse-esprit, pèse-liqueur, pèse-urine, pèsesel, pèse-sirop, pèse-nitre, pèse-lettre, pèse-bébé. Mais, comme on l'a déjà dit, RÈGLE À CALCUL(ER) est un mixte morpho-fonctionnel, ce qui constitue peut-être un compromis optimal entre une dénomination morphologique homogène et une dénomination fonctionnelle homogène. Pour ce qui est des définitions (et non plus des dénominations), 
l'importance de combiner ces deux types a été bien dégagée par les «travaux pratiques» présentés dans de Virville et alii (1977). Mais, dans une bonne mesure, une dénomination est le résumé sélectif et lacunaire d'une définition. Il est paradoxal de constater que la diffusion de cette dénomination, où l'importance de la maison Tavernier-Gravet a dû jouer un rôle majeur, conduit la France, si longtemps rétive devant l'instrument, à imposer le terme qui est le sien plutôt que le terme de type anglais. Quant aux dénominations sur le type REGLE LOGARITHMIQUE, elle sont en revanche peu représentées relativement à ce que l'on aurait pu attendre, tellement peu que cela ne semble plus représentatif.

Troisièmement, il ne semble pas exister de différences portant sur l'organisation thématique signalée plus haut comme envisageable a priori. On notera au passage que seul le slovaque dénomme la règle à calcul comme CALCULATEUR, et cela alors même que, en technologie, si nous avions à classer la règle à calcul, nous la rangerions sans hésiter sous le genre des calculateurs, taxon optimalement informatif (ni trop général ni trop particulier). Nous la rangerions donc dans la même catégorie que les calculi, les quipus andéens et leurs analogues chinois, les tailles sur bâton de bois, les bâtons à calculer chinois et leurs successeurs japonais et coréens, les jetons, l'abaque, sa variante chinoise le suanpan (parfois traduit en français cadran à calculer et en anglais par countingframe) et japonaise le soroban, les bâtons de Neper et leur postérité sur cylindres, les machines conçues par Pascal, Leibniz, Babbage et bien d'autres, les analyseurs harmoniques ou différentiels, les machines à calculer mises au point à partir du XIX ${ }^{\mathrm{e}}$ siècle (la machine inventée en 1820 par Charles Xavier Thomas, l'arithmomètre d'Arthur Burkhart, la machine de Léon Bollée, etc.), ou les machines à calculer électroniques contemporaines. Le slovaque étant isolé, nous voyons qu'un terme n'est donc pas nécessairement construit suivant un principe de développement taxonomique qui voudrait que l'on donne d'abord le genre puis l'espèce (dans le style des termes de botanique et de zoologie). Toutefois, nous verrons l'intéressant exemple d'une dénomination en espéranto plus loin, à comparer au slovaque.

Quatrièmement, comme attendu, très peu de dénominations incluent règles au pluriel, ou une expression duelle comprenant double, malgré des précédents comme l'anglais a pair of compasses. Les rares cas attestés sont obsolètes. Faut-il ici imaginer la concurrence et l'obstacle des règles = menstrues dans bien des langues de la famille indoeuropéenne, bien que l'objet ait été utilisé presque exclusivement par des hommes? De tels interdits par conflit homonymique avec un terme émotionnellement marqué ne sont pas à rejeter par principe, comme me le montre la réaction de telle femme se refusant à l'emploi de serviette et n'utilisant que cartable (et cf. le rooster des Américains pour cock, tabou à cause de son sens sexuel). En tout cas, il est assez vraisemblable que le filtre lexicogénétique formel (voir plus loin) défavorise les termes pluriels, malgré l'existence de contre-exemples comme ciseaux.

\section{2) FAITS NOUVEAUX NON PRÉVUS PAR LE MODĖLE}

Cependant, des faits nouveaux étaient absolument imprévisibles par notre modèle $\mathrm{n}^{\circ} 1$, trop grossier notamment parce que insuffisamment informé des réalités historiques, à savoir ces schémas supplémentaires obtenus à partir de diverses langues et à partir de l'anglais et du français ancien:

- PLANCHETTE / BÂTON / BAGUETTE À CALCULER (hongrois ${ }_{1}$, finnois, allemand $_{2}$, danois, norvégien, suédois, espéranto $\mathrm{O}_{2}$ );

- PLANCHETTE LOGARITHMIQUE (hongrois ${ }_{2}$ );

- BÂTON / PLANCHETTE Â MESURER (†anglais ${ }_{5}$, hindi $_{3}$ );

- RĖGLE D'INGÉNIEUR (arabe 3 ); 
- RÈGLE(S) PROPORTIONNELLE(S) (†anglais 3 , †anglais ${ }_{4}$, †français ${ }_{3}$ );

- MESUREUR À CALCULER (espéranto ${ }_{5}$ );

- COULISSEAU (hindi ${ }_{2}$ );

- GUNTER À COULISSE (†anglais 6 );

- RËGLE DE SOHO (†anglais 7,8 ).

Occupons-nous d'abord des deux premiers.

Notre schéma définitionnel $n^{\circ} 1$ était: (1) RÈGLE(S) (2) COULISSANTE(S) (3)

POUR CALCULER (4) AU MOYEN DE LOGARITHMES. Prisonnier des données initiales et de la construction des termes retenus, il n'avait pas pu pousser la décomposition sémique assez loin, puisque RÈGLE = BÂTON / PLANCHETTE / BAGUETTE GRADUÉE. Nous avions tenu à tort RĖGLE comme un primitif, et effectivement la très grande majorité des termes comporte REGLE, ce qui s'explique bien, car visuellement, une règle à calculer ne ressemble à rien tant qu'à une règle graduée banale, et quand j'étais enfant, je ne voyais pas la différence (tout en m'interrogeant sur la bizarrerie des graduations et sur l'existence d'une réglette et d'un curseur), d'autant qu'un côté biseauté de l'objet mystérieux comportait aussi une graduation banale de 25 centimètres. Mais certaines langues résistent à ce moule, puisque le hongrois, le danois et le norvégien vont en deçà de REGLE. On peut considérer que l'une au moins des dénominations de la règle à calcul (hongrois ${ }_{2}$ ) présente un déficit sémantico-référentiel, car PLANCHETTE À CALCULER pourrait aussi bien désigner hors contexte le bâton à encoches qui servait autrefois à faire les comptes pour le pain acheté chez le boulanger, par exemple (le tally (stick) des Anglais); il y avait aussi des tallies employés par l'Échiquier anglais. Mais la précision «hors contexte» est capitale, un terme n'étant en fait compréhensible que dans ce que j'ai appelé un «cadre interprétatif», qui fait en particulier référence au milieu utilisateur. Ceci vaut pour les termes techniques encore bien plus que pour les autres lexies (voir de beaux exemples dans Quemada 1978). Seul le cadre interprétatif peut par exemple désambiguïser entre les différentes interprétations du suffixe -mètre (signalées dans Guiraud 1978: 67, 98, 101-104).

Nous voyons donc combien nous avions eu raison de proposer spéculativement ce que nous avions appelé «modèle $n^{\circ} 2$ », soit: (1) PLANCHETTE(S) (2) GRADUÉE(S) (3) EN ÉCHELLES LOGARITHMIQUES (4) POUR CALCULER. Si nous l'avions suivi plus hardiment, nous aurions pu prévoir a priori les cas du hongrois, du finnois, du danois, du norvégien et du suédois, ainsi que certaines variantes de l'allemand et de l'espéranto. Noter enfin que ces langues ont choisi des nominations avec PLANCHETTE 1. BÂTON / BAGUETTE (en toute rigueur, il faudrait évidemment distinguer ces signifiés, mais peu importe ici), et cela malgré le fait qu'elles disposent de termes pour règle (comme lineal en danois et linjal en norvégien).

Malgré tout, même avec le modèle $\mathrm{n}^{\circ} 2$, la troisième dénomination de l'arabe, qui semble être un terme informel du Liban (RËGLE D'INGÉNIEUR), demeurait imprévisible. Il s'agit d'une dénomination par mention de l'utilisateur typique, comme dans compas de charpentier, compas d'appareilleur, règle d'appareilleur (sur une planche de 1800-1801 au Musée National des Techniques, Paris), chaîne d'arpenteur (= anglais surveyor's chain / chain measure), équerre d'arpenteur, engineer's chain, mètre de couturière, engineer's rule / scale («a scale that is commonly of triangular cross section and has different decimal scales on its edges», dit le Webster's Third). Une variante en est le type de désignation par domaine d'application typique, comme dans boussole de marine, boussole de mine.

D'autres dénominations sont apparues dans la riche collection de termes anglais anciens. D'abord le sliding Gunter, manifestement issu par ellipse d'un non attesté et sans doute jamais formé *sliding Gunter line(s) / scale(s), en vertu d'une contrainte 
linguistique formelle, d'un «filtre économique», défavorisant les termes ternaires, trop lourds (nous avions soupçonné déjà qu'une autre de ses contraintes tendancielles défavorise les termes pluriels). Ce n'est pas à dire que des termes à trois composants ultimes soient inconnus, comme cathode-ray tube ou scanning electron microscope, mais ils sont défavorisés. Nous avons vu aussi rulers of proportion et double scale of proportion (rares termes pluriels), qui sont à rapprocher de termes du type règle logarithmique, vu les rapports entre les concepts de logarithme et de proportion. N'oublions pas que la règle de Gunter comptait déjà parmi ses dénominations: rule of proportion, line of proportion, règle de proportion, règle proportionnelle (à côté de termes plus vagues comme line of numbers). Dans la conception des instruments à calculer des $\mathrm{XVI}^{\mathrm{e}}$ et XVII ${ }^{\mathrm{e}}$ siècles, les notions de rapport (anglais reason, puis ratio, français raison), et la notion seconde de proportion, ont joué un rôle capital. Ne pas oublier que si l'on fait glisser la réglette d'une règle à calculer de sorte que son 1 soit en face du 2 de la règle, on pourra «lire» sur les deux règles les proportions $2 / 1=4 / 2=6 / 3=8 / 4=10 / 5$. Les logarithmes étaient conçus au départ dans cette connexion avec le concept de proportion, qui au surplus pouvait aller jusqu'à couvrir ce que nous appellerions «progression», comme le montre bien cette définition: «Les logarithmes sont des nombres en proportion arithmétique, correspondant à d'autres nombres en proportion géométrique, desquels ils sont appelés logarithmes» (dans Jacques d'Ozanam, Table des sinus, tangentes et sécantes et des logarithmes, 1685, cité dans Dhombres et alii 1987: 184). Comparer à la définition d'allure plus moderne dans l'Encyclopédie de Diderot et d'Alembert: «Nombre d'une progression arithmétique, lequel répond à un autre nombre dans une proportion géométrique».

L'importance de la notion de proportion pour certaines des dénominations, tant de la règle de Gunter que de la règle à coulissement, est l'exemple de ce que nous pourrions appeler une dénomination conservatrice, qui donne l'impression (au moins pour nous) d'être un peu inadéquate, ou pour le moins de sous-estimer la nouveauté du principe de construction de l'instrument. En effet, le concept de proportion est inclus dans le concept de logarithme, mais il est moins puissant, moins riche que ce dernier. Parler de règle(s) de proportion au lieu de règle(s) logarithmique(s) est donc, dans une certaine mesure, une dénomination d'attente, qui regarde encore vers le passé, et qui peut se comparer à compas de proportion (j'en rappelle l'importance). Les différentes règles logarithmiques utilisent bien le concept de proportion, tout comme le compas de proportion, mais avec un quelque chose en plus qui fait toute la différence. Ce que nous touchons là, c'est le fait que les termes explicitement construits peuvent rester attachés à des idées anciennes alors même que le concept véhiculé est nouveau. Le cas est sans doute assez fréquent. On en trouvera un autre exemple, à vrai dire encore bien plus éclatant, dans l'expression principe oxygine (sic), utilisée d'abord par Lavoisier pour désigner l'oxygène, où le mot principe renvoie conservativement, et par une ironie involontaire, à la terminologie de la théorie du phlogistique de Stahl, que le même Lavoisier s'appliquait pourtant à réduire à néant (sur ces termes, voir Thagard 1992: 40, 46).

Le terme gauging-rod (= bâton à jauger / mesurer), à comparer à la première définition inadéquate de l'OED donnée plus haut ( $« A$ mathematical gauging or measuring instrument...»), constitue une appellation à la fois trop spécialisée, voire inexacte quant à la fonction (gauging) et trop imprécise (rod) quant à la morphologie. Il pourrait s'appliquer aussi bien à un appareil de mesurage linéaire. La même observation vaut pour un des termes du hindi (à vrai dire quasiment inusité). Sur Soho rule / scale, voir plus loin. Enfin, on peut constater que le rulers of calculation pour la règle de stade 2 (l'instrument d'Oughtred) n'a pas été réappliqué à la règle de stade 3 (type Bissaker et Partridge), alors qu'elle aurait pu l'être. Il semble donc bien que ce soient les Français qui l'aient indépendamment réinventé pour l'appliquer à la règle de stade 3 . 
Ne négligeons pas la possibilité de devoir considérer plusieurs schémas définitionnels, contrairement à ce que les brèves notations de Pottier laissaient attendre. On voit sur l'exemple de la règle à calcul que certains de ces schémas sont pratiquement constructibles a priori mais que d'autres sont imprévisibles. Comme chaque schéma définitionnel est susceptible de générer plusieurs représentations sémantiques, et qu'au surplus une représentation sémantique primitive est elle-même susceptible de subir une transformation tropique (comme la métaphorisation: voir plus loin) pour donner une représentation sémantique dérivée, on comprendra aisément le foisonnement terminologique possible. C'est ce foisonnement terminologique que nous allons examiner plus avant dans la section suivante.

\section{3) AUTRES CONCLUSIONS} négative.

Quelques autres conclusions annexes se dégagent, qui sont souvent sous une forme

Premièrement, aucun des termes n'est figuré, et spécialement métaphorique. Une dénomination métaphorique sexuelle aurait pu, à l'extrême rigueur, être imaginée $a$ priori, vu l'existence d'une réglette mâle coulissant dans une règle femelle. Il est vrai que si l'on parle de «partie mâle» et de «partie femelle», de «prise mâle» et de «prise fernelle», l'assemblage d'une partie mâle et d'une partie femelle n'a pas de nom, et pour cause, puisque cet assemblage n'a pas d'intérêt technique particulier. En revanche, partant du modèle de systèmes coulissants comme la valve tiroir (ou soupape à tiroir), on aurait aisément pu imaginer un *tiroir à calculer, ou un *tiroir logarithmique, dont le polonais est du reste extrêmement proche (tiroir étant un des sens de suwak), bien qu'en polonais, suwak ait pris le sens technique de coulisseau / curseur par métaphore déjà lexicalisée avant la constitution du terme. L'idée de coulissement se retrouve dans un des deux termes islandais, celui qui est formé à partir de stokkur, qui a pris le sens de plumier (dont le couvercle coulisse). On sait au reste que certains instruments ont des désignations métaphoriques, soit devenues opaques (exemples dans Thoiron 1994), soit explicites, comme l'antenne de radio appelée rabbit ears, la machine à mesurer type col de cygne (Aubert 1991 : R 1215, 15). Plus ludique encore est, parmi les compas, celui qui s'appelle en français maître à danser ou maître de danse (compas d'épaisseur dont les branches évoquent les jambes et les pieds d'un danseur) ou le compas assez proche appelé huit-dechiffre(s) (= figure-of-eight calliper / hour-glass calliper, selon deux variantes métaphoriques). Par ce qui est peut-être une amusante rétroaction de renforcement entre l'objet et ce qu'évoque sa dénomination, on connaît des maîtres à danser dont les branches prennent des formes explicites de jambes et de mains (Feller et Tourret 1970, $n^{\circ} 4$, en face de la p. 19), et même l'un, plus fantaisiste encore, «dont une partie des branches en $\mathrm{X}$ prend la forme de jambes féminines ornées de jarretières» (Velter et Lamothe 1977: 114-115, avec la photo discrètement polissonne); garder à l'esprit que les compas étaient souvent fabriqués par les mêmes artisans qui les utilisaient ensuite. Ces cas ressemblent aux cas de transferts de sèmes additionnels dans la métaphorisation (Boisson 1985).

Deuxièmement, aucun des termes courants n'inclut le nom de l'inventeur ou d'un des inventeurs, aucune n'est une dénomination éponymique: on aurait pu imaginer par exemple un *Partridge's rule sur le modèle de Gunter's line ou Napier's bones. Serait-ce que l'éponyme (Gunter, Oughtred, Partridge ?) est moins aisé à choisir? Faut-il considérer que Gunter's line a paru très mal approprié une fois découvert le principe ergonomiquement fondamental d'un coulissement? Cependant, on a vu attesté le terme de sliding Gunter, qui n'a pourtant guère connu de succès, alors qu'il n'était pas mal constitué. Les dénominations éponymiques ne sont pourtant pas rares: que l'on songe par 
exemple, dans des domaines connexes, à nonius, vernier, réglettes de Genaille (voir d'Ocagne 1905: 14; Baxandall 1926: 12; Marguin et alii 1990:38), Dunlop calculators (Baxandall 1926: 11), Cuisenaire rods, Johansson block, radio latino (ou trigonum, instrument d'arpentage mis au point au XVI e siècle par Latino Orsini: voir Demoriane 1960: 98), quartier de Davis (Davis's quadrant, instrument de marine), Gunter quadrant. Dans d'autres domaines, on a les corps désignés par leurs inventeurs dans l'alchimie et l'ancienne chimie (Guiraud 1978: 73), les dénominations anatomiques (trompe d'Eustache), et des termes techniques comme: étau Murinais, thermomètre de Bernoulli । Réaumur / Roberval, aréomètre de Nicholson, pile volta / voltaïque, Edison battery, Davy lamp, Geiger counter, Van de Graaff generator, Wilson chamber, Crookes tube, Wimshurst machine, et même le jadis célèbre Skinner box, plus les venturi, saxophone, saxhorn, bélinographe et autres draisine / draisienne, pascaline, micheline, michauline (= draisienne à pédales de Pierre Michaux), bessemer, diesel.

Cependant, on connaît des dénominations de ce type, sinon pour la règle à calculer en général, du moins pour telle ou telle variante ou sous-type: d'où règle Lenoir-Gravet, règle Mannheim, règle Rietz, règle Péraux, règle Beghin. Mais il semble s'agir plus de désignations technico-commerciales (semi-publicitaires, pourrait-on dire) que de désignations courantes. Personne, $\mathrm{j}$ 'imagine, n'a eu tendance à dire «Sortons notre (règle) Mannheim» au lieu de recourir au terme générique. Les noms de sociétés fabricantes ne sont pas non plus utilisés, chose qui se produit parfois, comme dans Frigidaire (alors qu'on dispose de frigo < frigorifique < appareil frigorifique), ou comme dans Post-It (personne ne disant removable self-stick notes $=$ notes adhésives repositionnables $=$ notas de quita y pon = biglietto autoadesivo rimovibile = Haft-Notizen = autokóllêtes sêmeiôsis, si judicieux que soient ces termes), ou encore dans la dénomination familière (propre à une entreprise?) que j'ai entendue, un fen pour un Fenwick = un chariot élévateur.

Troisièmement, un seul des termes inclut une spécification d'origine géographique, soit Soho rule, dont on a vu les précautions d'interprétation qu'il fallait lui appliquer et le statut d'usage un peu ambigu. On n'a pas eu de *règle anglaise (cf. règle lesbienne, un peu plus bas). Mais il faut dire que ce genre de dénominations, les dénominations de provenance (toponymique, ethnonymique), n'est pas très répandu, les exemples me venant immédiatement à l'esprit étant d'abord, pour rester dans un domaine connexe: Lesbian rule ( a mason's ruler, made of lead, which could be bent to fit the curves of moulding» dit l'OED ); cercle hollandais (= cercle d'arpentage, un instrument de géodésie); compas à l'allemande; quadrans judaicus (invention de l'astronome Jacob ben Machir ibn Tibbon, adoptée par les marins). Dans des domaines autres, on peut penser aussi à clé anglaise, Leyden jar (bouteille de Leyde), Leyden battery, Magdeburg hemispheres, charrue Brabant, établi à l'allemande, presse allemande, scie allemande (trois termes de menuiserie: Nosban 1873: 65, 70, 97). Ces termes d'origine ne deviennent raisonnablement courants — au moins pour les binaires - que pour des instruments de musique (cor anglais, French trumpet), des étoffes et tentures (velours de Gênes, Soho tapestries), des colorants, substances chimiques diverses, métaux (Epsom salts, blanc d'Espagne, bleu de Lyon, verre d'Angleterre, fonte Cleveland), etc. (un petit nombre de ces exemples sont repris de Quemada 1978).

Quatrièmement, aucun des termes n'est un mot unique «savant» à composition grecque ou gréco-latine, du type arithmomètre ou logocanon ou un imaginaire mais théoriquement bien formé *logarithmocanon, encore que son trimorphémisme soit vraisemblablement un handicap. En incidente, je noterai par curiosité le lapsus contenu dans un catalogue de la bibliothèque du Conservatoire National des Arts et Métiers, qui, dans le titre d'un livre, substitue à règle logarithmique un règle logarithmétrique fantaisiste... 
Cinquièmement, aucun des termes ne comporte un générique de faible niveau dans la hiérarchie conceptuelle de la famille d'objets calculateurs, comme le terme de multiplicateur (formant, mettons, un *multiplicateur logarithmique), lequel n'était pas inimaginable, l'instrument servant au départ essentiellement à effectuer des multiplications.

Sixièmement, enfin, sémantiquement on n'observe presque nulle part de dénomination unaire, sauf dans un des termes du hindi (glosable COULISSEAU), mais qui, dans les faits, est pratiquement inusité. Nous n'avons pas non plus de dénomination ternaire optimale, comme sur le schéma RÈGLE(S) COULISSANTE(S) À CALCUL, par exemple, ou COULISSEAU LOGARITHMIQUE À CALCUL. Toutefois, le terme utilisé par Seth Partridge est bel et bien un terme ternaire, mais d'un autre type, puisqu'il s'agit de double scale of proportion, dont il est significatif qu'il soit resté isolé et sans lendemain. Le souci de brièveté suffit à expliquer cet avortement, le terme devant manifester un compromis entre des considérations d'économie et des considérations d'adéquation, l'optimal étant en l'occurrence binaire. Les exceptions se rencontrent dans les disciplines où la taxonomie joue un rôle central voire organisateur, comme la zoologie et la botanique, où l'on trouve par nécessité des termes ternaires du type Homo sapiens neanderthalensis - et la chimie, encore qu'on y trouve souvent par commodité des désignations usuelles très abrégées. On peut aussi trouver des désignations lourdes dans des textes à forte contrainte de sous-catégorisation, comme les catalogues de fabricants, mais on sait que les dénominations commerciales ne témoignent pas en général de l'usage courant des utilisateurs des produits. De toute façon, si le langage ne servait qu'à l'expression ou la cogitation, des termes lourdement explicites seraient acceptables, mais puisqu'il est aussi un système de communication, des considérations d'économie interviennent, l'excès d'explicitation étant coûteux, et, comme on l'a dit, un filtre lexicogénétique formel défavorise ce genre de termes complexes (voir l'exemple caractéristique de Post-It, plus haut). On doit donc opter pour un optimum, qui semble tendanciellement être binaire. À l'inverse, une dénomination sémiquement unaire (au degré de décomposition pertinent) est difficilement concevable, car elle courrait le risque d'une ambiguïté insupportable, au moins comme dénomination standard pleine, sinon comme abréviation informelle, comme si on disait: *règle, *coulisse.

De toute manière, nous devons mettre l'accent sur ceci : des inférences naturelles au vu des connaissances courantes permettent vraisemblablement de prévoir le trait $\grave{A}$ CALCUL à partir de LOGARITHMIQUE, les logarithmes n'ayant normalement d'intérêt pratique qu'aux fins de calcul (il peut en aller différemment pour des mathématiciens professionnels, ce qui est une autre affaire). Ces règles d'inférence - développables à la demande, en quelque sorte - jouent certainement un rôle majeur dans la construction des termes, et qui demanderait à être examiné systématiquement. Elles constituent elles aussi une des composantes de ce que j'ai appelé le cadre interprétatif, et elles contribuent à déterminer l'existence du filtre lexicogénétique formel. Au reste, on en voit à nouveau l'effet conjoint dans un fait comme celui-ci. Thoiron se demande si le trait sémantique GRADUATION ne pourrait pas apparaître dans telle ou telle langue. Nous avons vu qu'il n'en est rien en général (la seule exception étant le terme archaïque Gunter's scale). L'explication est assez simple. (1) Une règle d'inférence du cadre interprétatif permet d'inférer GRADUATION de RËGLE et de CALCUL, ou de LOGARITHMIQUE. (2) Si GRADUATION se manifestait formellement, il devrait s'incarner par un troisième composant du terme, qui serait alors du type: RÈGLE GRADUÉE À-CALCULER ou RĖGLE GRADUÉE LOGARITHMIQUE (il faut bien que la notion de GRADUATION soit appliquée à un support). Or le filtre lexicogénétique formel interdit pratiquement des termes à trois composants, peu économiques. Tout cela implique également que les rermes sont ininterprétables (ou ne sont décodables que vaguement) en dehors d'un 
système d'organisation des connaissances. Pour le terminologue, cela signifie aussi que la terminologie est une discipline-carrefour qui touche à la fois à la linguistique (en premier lieu, bien sûr), à l'étude des systèmes conceptuels comme les mathématiques, par exemple, à l'étude des systèmes d'inférences courants qui relient des entités dans des complexes fonctionnels, ainsi qu'à la technologie.

Il va de soi que les règles d'inférence ont leurs limites, que, de toute manière, elles ne sont pas associables a priori à un terme et qu'ainsi ce terme peut être pratiquement ininterprétable. Par exemple, supposons le jeu de langage suivant: des informateurs vivant sous un climat où l'arc-en-ciel est inconnu essaient de deviner ce que peut être le référé des termes désignant l'arc-en-ciel dans diverses langues. Éliminons les termes simples, pratiquement inanalysables (et assez courants, comme en grec, en tchèque, en bouroushaski, en piro), ainsi que les termes usités dans des cultures où l'arc-en-ciel est conceptualisé comme un serpent, ou un pont, ainsi qu'il est fréquent, ou bien les roue de St Bernard / St Martin et courroie de St Barnabé qu'on trouve en franco-provençal. Reste que nos observateurs, confrontés à un ensemble de termes raisonnablement «techniques» comme arc-en-ciel, rainbow, Regenbogen, le terme hébreu, qui dit «arc-en-nuages», l'italien arcobaleno (baleno = éclair), seront bien en peine de comprendre de quoi il s'agit, et cela sans même parler du arco iris (espagnol, portugais), avec emprunt au grec.

Septièmement, on n'observe aucun cas d'emprunt pur et simple, comme computer à date récente ici ou là (pour un Français, le terme comportant le radical comput_ est au moins aussi bien fait qu'ordinateur, quoique, assurément, l'ordinateur se soit révélé utile à bien d'autres choses qu'à la computation au sens restreint). Un principe général opère vraisemblablement, savoir qu'un mot composé s'emprunte moins aisément qu'un mot non-composé, même polymorphémique, car une traduction-calque est ici la solution évidente.

Huitièmement, nous avons constaté l'existence en Angleterre d'un foisonnement de termes qui sont restés en compétition jusqu'au XVIII' siècle, et dont seuls ceux qui sembleraient être les plus anciens (sliding rule peut-être en premier, et slide rule) ont survécu et sembleraient avoir dominé dès le départ. Il s'agit probablement d'un cas assez courant en terminologie: on verrait ainsi aisément comment la multiplicité des termes relatifs à la bicyclette et ses ancêtres au XIX ${ }^{\mathrm{e}}$ siècle s'est réduite en français au terme standard bicyclette et au terme familier vélo, issus de deux étymons différents, tandis qu'en anglais on a bicycle et bike respectivement, le deuxième terme étant une abréviation du premier. Il doit s'agir d'une situation qui s'explique doublement. Il s'agit en partie du fait que chaque inventeur responsable d'une amélioration parfois minime, chaque fabricant, a tendance à essayer d'imposer son terme par fierté d'auteur. Linguistiquement, ce buissonnement terminologique n'est évidemment pas viable à terme, quoiqu'il puisse subsister assez longtemps (mais il faudrait pouvoir établir des statistiques sur la fréquence d'emploi des divers termes et l'évolution de ces fréquences, et l'on verrait sans doute que certains ont toujours été d'emploi minoritaire). Noter pourtant qu'en espéranto, il est plus tolérable, parce que la systématicité assez grande de la lexicogenèse autorise des décodages faciles. Le buissonnement terminologique est aussi lié au fait que, dans un stade initial, l'objet se présente non pas comme un être isolé, mais comme membre d'une famille de variantes qui appellent des noms plus ou moins différents, cela jusqu'à ce que les variantes moins satisfaisantes finissent par céder la place à un modèle standard, classique, qui est le meilleur modèle, ou au moins le modèle optimal par son compromis entre des contraintes diverses, comme la bicyclette dite d'abord safety bicycle, ou la règle à calcul type Mannheim. Une uniformisation «orthonymique» de la nomination doit alors correspondre à la victoire de cet «objet canonique». 
Neuvièmement, la règle de stade 4 (système Mannheim) n'a pas de nom usuel différent de la règle de stade 3 , ce qui se justifie par le fait que l'innovation du curseur n'est pas un élément essentiel de la règle à calcul, même sous sa forme achevée. De même encore, ce n'est que dans une sous-catégorisation (conduisant à une analyse sémantique fine sans incidence sur les dénominations usuelles) que l'on aurait à envisager une [[RÈGLE À CALCUL] [À [CURSEUR À LOUPE]]].

Enfin, d'une manière générale, nous constatons que les dénominations ne sont que partiellement prévisibles a priori. J'en veux pour preuve supplémentaire le petit test suivant. Ayant demandé à un spécialiste de l'espéranto, au surplus spécialement compétent puisque mathématicien de formation, François Lo Jacomo, comment se dit règle à calcul en espéranto, celui-ci m'a indiqué comme formation personnelle («si je devais le traduire, j'utiliserais sans doute...») le terme de linia kalkulilo (= calculateur linéaire, -il-étant un suffixe instrumental), avec le commentaire suivant: «calculateur linéaire, compte tenu qu'une règle se traduit par liniilo = instrument pour faire des droites (linioj), avec le même suffixe instrumental que dans kalkulilo». Tous les informateurs espérantistes mathématiciens consultés par Lo Jacomo ont considéré cette formation comme compréhensible, quoique non optimale (leur préférence allant à glitkalulilo, exprimant le coulissement).

Or, ce qui est très intéressant dans cette proposition terminologique de Lo Jacomo, c'est qu'elle réalise l'image en miroir du terme français, par renversement de l'orientation thématique. Lo Jacomo, comme mathématicien, a choisi la solution logique évidente de catégoriser la règle à calcul comme un CALCULATEUR, le seul parallèle attesté dans notre échantillon de langue naturelles étant, rappelons-le, le cas du slovaque. Mais là où le slovaque fournit la représentation sémantique CALCULATEUR LOGARITHMIQUE (réalisé avec mention du principe mathématique dans le modificateur), l'espéranto ici proposé est CALCULATEUR LINÉAIRE / À RÈGLE (réalisé avec mention de la morphologie dans le modificateur).

Qui plus est, le terme qui semble préféré par les mathématiciens espérantistes, glitkalulilo, a comme représentation sémantique CALCULATEUR À GLISSEMENT, qui n'est autre que le renversement de COULISSEAU À CALCULER (allemand, néerlandais 2 ).

Corrêlativement, se trouve posé le problème de l'adéquation du terme, à propos de quoi je citerai Thoiron:

Une question fondamentale dans le cadre de la didactique d'une discipline est celle de la transparence (on pourrait dire motivation) du signifiant. Un signifiant est d'autant plus transparent qu'un plus grand nombre des traits conceptuels du concept correspondant est intégré dans le processus de nomination. L'exemple du français LOUPE permettra d'illustrer ce point. Le signifiant ne dit rien du concept. En revanche, l'équivalent anglais magnifying glass («verre qui grossit») donne accès directement à deux traits conceptuels du concept LOUPE: la matière et la fonction [...]. La recherche de la motivation, ou de la transparence, est un but à poursuivre dans le cas de la néologie. On peut d'ailleurs évaluer les créations terminologiques selon ce critère.

Et comme le dit Guiraud à propos de la chimie (1978: 5-6) : «Un mot comme sulfate de cuivre nous renseigne sur la nature chimique de la chose. Le terme entre, par ailleurs, dans un système de classification qui oppose sulfate à sulfite, sulfure, sulfone». Pour répéter Guiraud, on a là des termes qui, en eux-mêmes, donnent une certaine information sur le référé, et cela conformément aux rêves des divers concepteurs de langues universelles a priori du XVII ${ }^{\mathrm{e}}$ siècle, comme les Wilkins et les Leibniz. Ce dernier avait déjà observé qu'un mot comme phosphore fournit une indication sur l'objet désigné. 
Mais avec le cas de la règle à calcul, comme l'indique programmatiquement Thoiron dans l'article dont le présent est un développement, nous voyons que l'objet est d'autant mieux atteint, circonscrit, que nous rassemblons un plus grand nombre de ses dénominations; c'est ce que pressentait déjà très clairement un philosophe chez qui la notion de "point de vue» joue un rôle capital, Leibniz toujours, déjà cité en exergue, et ainsi paraphrasé par un de ses commentateurs modernes: «Cependant on ne parvient pas toujours à dénombrer toutes les idées simples qui forment un concept; alors en rassemblant autant que l'on pourra de définitions nominales, chacune dégageant tel ou tel caractère, on multipliera les points de vue pour pousser plus loin la décomposition [...]. Du même objet plusieurs expressions sont possibles qui le représentent comme d'autant de points de vue» (Belaval 1952, 1989: 126, 131).

Il est loisible de reprendre toutes les dénominations de la règle à calcul dans cette perspective, en comparant les représentations sémantiques correspondantes au schéma définitionnel associé et en jaugeant l'adéquation des termes suivant certains critères indépendants qui resteraient à spécifier, permettant par exemple de préciser ce qui pourrait constituer une «saillance conceptuelle» (sur la saillance, voir Le Ny 1979: 190 sqq.). Je ne l'ai fait ici ou là qu'en passant, mais une expérience possible consisterait à prendre un groupe de sujets et à leur soumettre des dénominations diverses pour une entité, puis d'observer leurs réactions. En tout cas, il est clair que, a priori, un terme fondé sur un trait fortement contingent, voire carrément fortuit, comme Soho rule, sera estimé moins approprié qu'un terme construit sur des traits nécessaires, définitoires, comme ceux du russe ou du français.

On terminera par quelques autres faits intéressants touchant à la diffusion vraisemblable des dénominations, en relation avec la diffusion de l'objet, longtemps fabriqué, on l'a vu, seulement en Angleterre, en France et en Allemagne. L'anglais demeure presque aussi isolé qu'auparavant, puisque seuls lui ressemblent: (a) les emprunts directs du hindi (le seul des trois termes hindis effectivement utilisé dans la pratique) et du bengali; (b) le terme irlandais et un des deux termes gallois, calqués sur lui, l'Irlande et plus encore le pays de Galles étant fortement soumis à l'impact anglais ; (c) une des deux variantes principales de l'arabe - l'arabe semblerait disposer d'un terme venu du français et d'un autre venu de l'anglais, l'anglais et le français constituant les deux modèles terminologiques pour cette langue. De même, chose remarquable, un des deux termes gallois est du type règle à calcul.

Ces données méritent attention, car, contrairement à ce qu'on aurait pu attendre, l'anglais n'a pas suscité une vaste postérité de traductions-calques. Voilà un cas hors norme, car usuellement l'avance technique d'une nation se traduit par l'exportation de ses termes: c'est pourquoi la terminologie minière en Europe est massivement d'origine allemande, les termes nautiques français viennent souvent du néerlandais, et ainsi de suite (Gille 1978a: 91). Ce cas renferme aussi une leçon pour les études de terminologie historique : il suggère que si nous étions ignorants de l'origine de l'objet, nous ne serions pas en mesure de la reconstituer au seul témoignage des dénominations actuelles, lesquelles, par leur distribution, laissent attendre faussement une invention française; ce qui nous incite à la prudence dans le recours aux données linguistiques dans des situations où notre information est très lacunaire. Les diffuseurs ne sont pas nécessairement les concepteurs, et le foyer d'irradiation ne coöncide pas nécessairement avec le lieu de l'invention. Cette précaution de méthode doit d'autant plus s'appliquer que les innovations sont anciennes, de circuit complexe et éventuellement «réinterprétées», comme dans le cas des meules à ocre réutilisées comme meules à céréales dans un cadre néolithique, une fois l'agriculture apparue, si bien qu'un même terme aura pu désigner d'abord les unes puis les autres (Blažek et Boisson 1992). 
En revanche, la même remarque ne s'applique pas à l'allemand, du moins pour l'une de ses deux dénominations (celle qui est sur le schéma BAGUETTE À CALCULER), qui semblerait avoir irradié vers les pays nordiques (d'où les termes danois, norvégien et suédois, ce dernier ayant dû servir de modèle-relais pour une diffusion seconde vers le finnois), ainsi qu'en hongrois; tout ceci s'expliquant bien par l'influence technique et culturelle de l'Allemagne sur ces pays. Pour autant, le rayonnement allemand se révèle plus discret qu'il n'était prévisible. Le russe a influé au moins sur le letton (peut-être sur le tchèque?).

Il est clair aussi que la dénomination française usuelle (règle à calcul) a joué un rôle de modèle terminologique, de générateur de calques pour toute une série de langues, soit dès le départ, soit peut-être en se substituant à une dénomination autre plus ancienne. Ce modèle détermine une cascade, comme dans le français, générant l'espagnol, qui génère le basque. De la sorte, nous devons avoir conscience que la prédominance des dénominations sur le schéma RĖGLE À CALCUL ne prouve pas nécessairement la saillance particulière des traits sélectionnés, qui se serait imposée indépendamment aux langues diverses. En effet, il serait tentant de définir un ensemble de traits saillants, centraux, entrant dans la conceptualisation prototypique de la règle à calcul. On peut estimer que l'ensemble des traits est hétérogène et que chaque trait se voit affecter d'un poids propre. La saillance joue donc en principe un rôle important qui se manifeste dans la dénomination. Mais, comme nous le voyons, à cause de la circulation des mots avec les choses qu'ils désignent, nous sommes ici tenus à la prudence. Il faut combiner les explications psychologiques (saillance) et les explications ressortissant de l'histoire de la technique. Le dosage et l'interaction entre ces explications ne sont pas faciles à opérer. Dans notre cas, faudrait-il supputer que si la dénomination anglaise a été si peu imitée, c'est parce que, d'une certaine manière, elle était peu «naturelle». Il serait alors plus normal de catégoriser la règle à calculer comme un type particulier de la famille des objets appelés règle, et non pas, comme on aurait pu s'y attendre aussi, comme un type particulier de calculateur. Faut-il faire la part de contraintes historiques dans le choix des modèles (et même, à la limite, de «modes» dénominationnelles)? Faut-il imaginer que, à une époque où la classe des calculateurs restait encore pauvre et peu répandue, il était plus naturel de catégoriser l'objet par référence à une autre classe alors bien connue, celle des règles. Toutes choses égales d'ailleurs, une classe d'objets riche et largement répandue constituerait un attracteur terminologique plus puissant qu'une classe d'objets pauvre et rare.

D'un autre côté, on ne peut manquer d'être frappé par la différence qui existe parfois entre des langues voisines. Comparez ainsi le tchèque et le slovaque - le dernier s'étant peut-être distingué du premier par nationalisme ostentatoire, en créant un terme qui trouve son parallèle dans une des variantes de l'espéranto. Voyez plus généralement l'éventail des dénominations parmi les langues slaves, s'opposant à l'homogénéité remarquable des langues romanes, très probablement imitatrices du français. Par ailleurs, noter que, en bonne logique lexicogénique, le grec s'est spontanément constitué un terme qui ressemble (analytiquement) au synthétique logocanon de Gunter.

Un problème pour lequel je n'ai aucune solution est celui de savoir s'il existe des appellations alternatives familières de la règle à calcul (autres que l'évidente ellipse règle), comparables à zinc $=$ avion, bécane mis pour toutes sortes de machines, y compris, récemment, l'ordinateur (voir Quemada 1978: 1156). 


\section{CONCLUSIONS}

Nous pouvons maintenant rassembler l'ensemble assez volumineux des types de dénominations trouvées en diachronie et en synchronie pour la règle à calcul, en réordonnant les types, et, par commodité, en commençant par les types prédominants.

Voici les 17 types de dénominations recensés:

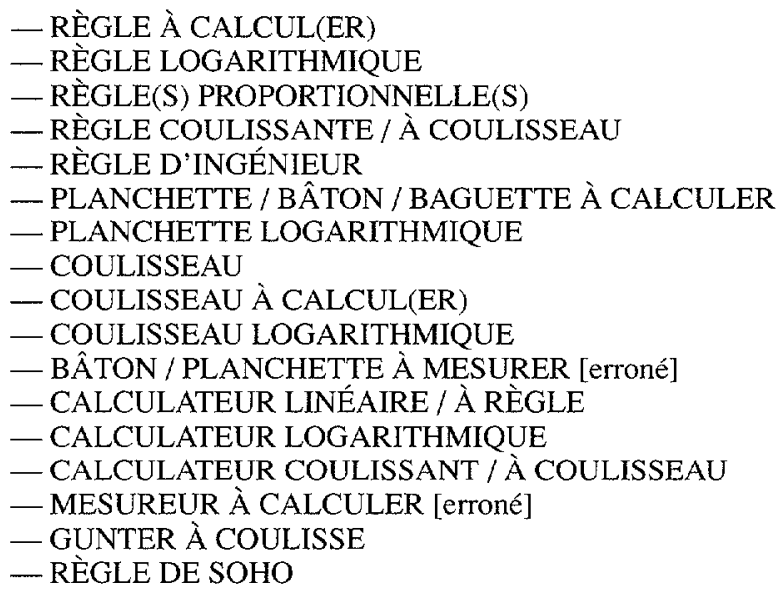

Distributionnellement, les conclusions sont: (1) Si l'on se fonde sur l'élément thématiquement dominant du composé, les dénominations par rattachement à une classe d'entités concrètes (RËGLE, PLANCHETTE, BÂTON, COULISSEAU) prédominent nettement sur les catégorisations abstraites (CALCULATEUR, MESUREUR). (2) Le plus grand nombre de dénominations catégorise l'objet comme une RÈGLE, et parmi ces cinq classes de dénominations en RÈGLE, c'est RÈGLE À CALCUL(ER) qui prédomine largement dans la distribution des langues. (3) La notion de COULISSEMENT est assez fréquente, soit thématiquement dominante, soit thématiquement dominée. (4) En revanche, la notion de LOGARITHME est considérablement moins fréquente qu'on aurait pu le croire.

Des données tirées de notre échantillon étendu, on pourra s'autoriser à reconstruire informellement un «schéma définitionnel panlinguistique» du type : (I) Morphologie : (1) RÈGL; (II) Manipulation : (2) À COULISSEAU; (III) Utilisateur : (3) DESTINÉE TYPIQUEMENT AUX INGÉNIEURS; (IV) Fonction: (4) POUR CALCULER; (V) Principe théorique: (5) PAR LES LOGARITHMES. Ce schéma définitionnel panlinguistique constitue une approximation empirique de l'analyse du concept de la règle à calcul. Pour être plus rigoureux, $\grave{A}$ COULISSEAU fonctionne à la fois comme information morphologique et comme information indirecte sur la manipulation.

Montrons sans crainte de naïveté apparente par quelle procédure peut s'enrichir ce schéma à la fois puritain et flou, grâce à la prise en compte du «module inférentiel» du cadre interprétatif, boîte dans laquelle se trouvent des schémas d'inférence (valables pour toute une série d'objets techniques), et où l'on peut faire entrer les informations atomiques du schéma définitionnel pour en voir ressortir des informations supplémentaires. Les informations portées par les atomes (1), (4), (5) entrent dans le module inférentiel, lequel comprend un schéma d'inférence du type: «Si un objet de la classe morphologique des règles fonctionne en utilisant une ou plusieurs suite(s) numérique(s), alors ledit objet porte gravée l'image physique (ou graduation) de cette ou de ces suite(s) numérique(s)», lequel dépend de l'une des branches alternatives d'un méta-schéma d'inférence du type: 
«Si un objet met en œuvre un principe, alors ce principe s'y manifeste physiquement, soit dans la manipulation qui en est faite, soit par une trace physique». Ceci nous générera l'inférence morphologique $n^{\circ} 1$ : «La règle porte au moins une graduation logarithmique». À ce stade, on ne peut encore distinguer la règle de Gunter (stade 1, avec une seule graduation) et la règle à calcul proprement dite (stades 2 et suivants). Pour s'approcher du concept adéquat, il faut entrer à nouveau dans le module inférentiel en y injectant l'information portée par l'item (2) À COULISSEAU. Cet item contient par définition la proposition: «Il existe deux règles coulissantes». Cette proposition peut alors s'associer avec notre inférence morphologique $n^{\circ} 1$ pour prédire l'existence d'au moins deux graduations que l'on peut faire coulisser l'une en regard de l'autre (inférence morphologique $n^{\circ}$ ). De plus, nous pourrons faire un retour dans le module inférentiel par injection des informations portées par les atomes (1), (2) et (5) (plus les connaissances relatives aux logarithmes, qui font partie des connaissances encyclopédiques supposées) et confrontation avec un schéma d'inférence adéquat, ainsi qu'à la première branche alternative du méta-schéma d'inférence cité plus haut; ceci nous permettra d'affiner la caractérisation de la manipulation, qu'il serait oiseux de décrire par le menu. Mais ceci n'est possible que si l'on connaît les propriétés des logarithmes, ce qui n'est pas nécessaire pour une utilisation de la règle à calculer, comme dit plus haut. Tous les objets et machines conceptuels dont il est ici question ressortissent du cognitif. Autre est le statut du filtre lexicogénétique formel (comportant notamment deux règles probabilistes défavorisant respectivement les termes pluriels et les termes ternaires), lequel ressortit du linguistique entendu restrictivement.

Naturellement, les esquisses ci-dessus fonctionnent à partir du schéma définitionnel panlinguistique, et non à partir de la représentation sémantique du terme de telle et telle langue, qui en constitue un appauvrissement par l'intervention du filtre lexicogénétique formel. L'opération du module inférentiel s'en trouve nettement appauvrie, par exemple pour le français, puisque règle à calcul, avec sa représentation RËGLE À CALCUL, ne dit rien sur le principe théorique.

J'ignore quelle peut être l'utilité pratique ultime de telles entités et de telles procédures, mais ces jouets conceptuels nous permettent au minimum de poser avec plus de précision des problèmes de terminologie, et sans doute d'en faire surgir d'autres.

On trouvera dans l'appendice II une ébauche d'ouverture possible sur d'autres données relatives à la terminologie de certains objets techniques.

\section{J. QUELQUES INCERTITUDES THÉORIQUES FINALES}

Parmi les divers problèmes de fond que je n'ai fait qu'effleurer, figure celui des «traits», qui est apparu dès le début avec la mention du «modèle relationnel» de Pottier. De quelle nature sont ces traits? Il est fréquent de voir opérer une distinction entre des «traits de substance» et des «traits sémantiques». Sans examiner de près des problèmes épistémologiques épineux, le premier commentaire que je ferai là-dessus, c'est que l'expression «trait de substance» sans nuances ne me satisfait guère, car elle suggère l'extraction immédiate de caractéristiques inhérentes à l'objet considéré en soi. Sans même parler de la peine faite à un certain nombre de philosophes par une telle conception, le nombre de ces traits de substance est énorme; leur nature est variée autant qu'on peut l'imaginer. Au reste, c'est à se demander si la notion même de «trait» est adéquate. Même si elle l'était, seul un observateur omniscient, et sans aucune limitation dans le traitement de l'information, saurait en épuiser la liste. C'est ce que voit très bien Pottier (1992a: 65): 
Le référent physique propose à notre perception une infinité de possibles. Devant un arbre, je peux 'voir' le tronc, la forme ou la couleur des feuilles, les fruits, les fleurs, les oiseaux qui y sont perchés, les papillons posés... Mes habitudes culturelles, ma situation de communication et mon intention de discours me font sélectionner (focaliser) deux ou trois "traits référentiels' qui seront nécessaires et suffisants pour que je choisisse par exemple la dénomination arbre. Il est donc naturel que ces traits se retrouvent en partie dans l'analyse sémique.

Aussi l'objet doit-il être plutôt envisagé selon la conceptualisation qui en est faite (pertinente à tel objectif assigné), et ce mode d'appréhension négligera une quantité énorme de caractéristiques sans pertinence. Cette conceptualisation est ainsi fonction d'un point de vue. C'est aussi ce que dit Agazzi (1978), qui donne l'exemple du violon, «chose» à partir de laquelle se construisent des «objets» différents pour le luthier, l'acousticien, le spécialiste des vernis, le commissaire-priseur, etc.; la construction de ces objets selon des sélections de traits pertinents ne conduisant d'ailleurs pas à un phénoménisme agnostique quant au statut ontologique de ces objets. Cette façon de voir était déjà celle des auteurs médiévaux qui se transmettaient la conception «talia sunt subjecta qualia permittuntur a suis praedicatis». Dans une perspective voisine, Le $\mathrm{Ny}$ (1979) dit justement que la saillance des attributs est situationnelle, de sorte que, pour un piano, le trait «poids», normalement peu saillant, pourra acquérir une forte saillance dans certains contextes et pour certaines relations, comme celles qu'auraient des déménageurs avec un piano. Le fabricant de règles à calcul s'intéressera légitimement à toute une série d'attributs peu pertinents pour nous: finesse des graduations, lisibilité des graduations en fonction de la substance utilisée, degré optimal de frottement de la réglette, indéformabilité des matériaux employés (buis, métal, ivoire, plastique), et ainsi de suite. De sorte qu'en droit, on se demande si l'on n'est pas fondé à dire que l'ensemble des «traits de substance» d'un objet est infini. Cela est parallèle au fait qu'à n'importe quelle question, on peut faire toute une série de réponses logiquement correctes mais «impertinentes», comme fit cet élève qui, à la question «Louis XVI fut guillotiné en 1793. Quelles en furent les conséquences ?», répondit «Louis XVI mourut» - les recueils de «perles» sont pleins de ces réactions. C'est pourquoi je préférerais me borner aux «traits épistémiques» (les «traits conceptuels» de Thoiron 1994) sélectionnés par le point de vue, qui sont les seuls qui nous intéressent pour la dénomination. Je dis «trait épistémique» simplement pour négliger les connotations, ailleurs capitales, mais ici plus faciles à négliger, même si elles ne sont pas totalement absentes (dans la dénomination arabe «règle d'ingénieur», «ingénieur» ne saurait manquer de posséder une connotation forte).

Ceci posé, et pour revenir à notre problème initial, peut-on et doit-on établir une distinction entre «traits épistémiques» et «traits sémantiques»? On a vu que j'ai plutôt penché pour une indistinction de principe entre l'encyclopédie et le dictionnaire, ce qui rend floue voire à strictement parler inexistante une telle distinction (cela semblerait être un peu la solution de Desclés (1993), qui évoque des «primitives sémantico-cognitives»). Les traits sémantiques associés à un terme ne seraient en somme au mieux qu'une sélection de traits épistémiques, spécialement ceux qui correspondent à des propriétés douées de forte saillance - encore qu'on puisse objecter que la pensée, donc la manipulation des concepts, est possible sans langage, ce qui plaiderait éventuellement pour une distinction «conceptuel» vs. «sémantique». La solution suivant laquelle le sémème est une partie du concept est celle d'Arnaud (1987). Évidemment, si l'on est strictement saussurien, on tiendra à ce que le signifié soit établi de manière purement relative, différentielle, comme une «valeur» où les traits se définiraient par opposition. Au contraire, si la position vers laquelle j'incline est correcte, il faudrait comprendre mon terme de «représentation sémantique» comme indiquant simplement une sélection de traits épistémiques (noter au 
passage que Saussure ne distingue pas le concept ou notion et le signifié, pour la raison citée dans l'introduction).

Toutefois, bien se souvenir que la problématique des traits est indûment réductionniste et qu'il faut envisager des propositions telles que le schéma définitionnel, et non pas de simple liste de propriétés: je dirai donc vaguement que le concept, compréhensionnellement entendu, n'est pas réductible à une liste de propriétés et doit se concevoir comme un système où interviennent des propositions complexes, notamment, comme on l'a vu, à cause de la nécessité des inférences.

Bien sûr, il faut aussi reconnaître la force de la distinction entre Sinn et Bedeutung pour les logiciens, quoique dans le cas des objets techniques, on puisse soupçonner qu'elle ne joue guère de rôle. Au surplus, la distinction met typiquement sur le même pied des cas que l'observation réaliste des variétés d'emplois conduit à distinguer. (1) Les métaboles purement stylistiques évitant une répétition esthétiquement honnie, comme l'évêque de Meaux = l'auteur du $"$ Discours sur l'Histoire Universelle» = Bossuet. (2) La modulation conceptuelle, le même objet étant considéré selon diverses de ses propriétés, également définitoires, comme dans l'exemple à vrai dire très artificiel de triangle $=$ trilatère; la modulation conceptuelle est d'ailleurs assez dans l'esprit de Frege, pour qui le sens est un mode de présentation, une perspective, un éclairage du référé (Hottois 1989: $215,217)$. (3) La différenciation des signifiés due à une ignorance de l'identité du référé, comme c'est en fait le cas dans l'exemple premier de Étoile du matin $(=$ Lucifer $=$ Phosphorus $)=$ Étoile du soir $(=$ Vesper $=$ Hesperus $)$. Une fois acquise l'identité référentielle Étoile du matin = Étoile du soir = Vénus, le cas (3) se résout en une métabole.

Pour se résumer. Il est acquis depuis les stoïciens (Chrysippe) qu'il nous faut au moins trois entités. (a) On a le référé (par consistance morphologique, j'emploie ce terme plutôt que «référent» (cf. Hottois 1989: 209)). Dans un autre type de terminologie, on parle de denotatum (la classe des règles à calcul) et on distingue le denotatum (en langue) du référent (en contexte, en discours), cette règle à calcul particulière dans tel contexte. Par précaution généreuse, le statut ontologique de ce référé ou denotatum sera aussi divers qu'on voudra (réel, irréel, possible, etc.) dès l'instant qu'il est pensable, même ludiquement, et ainsi également dans les cas où il est empiriquement inexistant ou tenu pour tel, et dans les cas où il est logiquement contradictoire, donc y inclus les référés de Pégase, Don Quichotte, voire de cercle carré ou de le plus grand nombre. Il pourra désigner des «objets» comme kangourou; des phénomènes, comme l'arc-en-ciel, l'horizon ou le vent, voire le mirage; des processus ou comportements relatifs aux objets, comme courir; des idées, comme l'avarice; des fictions, ainsi qu'on l'a vu; mille autres choses. Je laisse ouverte la question obscure pour moi, je l'avoue, de savoir quel est le champ de ce référé: une fois acquis que, hors actualisation, kangourou ne désigne pas un kangourou particulier, kangourou désigne-t-il l'ensemble de tous les kangourous (mais alors à distinguer vraisemblablement de l'espèce biologique kangourou, véhiculant sa scolastique «kangourouité» dans son code génétique)? un membre quelconque de l'ensemble de tous les kangourous, censé être constitué d'individus interchangeables? le kangourou prototypique, le cas échéant associé à une image mentale (noter qu'il sera sans doute femelle, à cause de la poche, trait saillant, mobile à cause du mode de locomotion bondissant, autre trait saillant, et à certaines époques il aurait été boxeur)? (b) On a le signifiant, manifesté par diverses réalisations matérielles acoustiques, graphiques, voire iconiques. (c) On a un tertium quid, le signifié / le concept (ou notion).

Touchant (c), la question reste ouverte de savoir s'il convient d'en rester avec les stöiciens et Saussure à une indistinction entre signifié et concept, ou s'il convient de distinguer dans (c) le signifié (entité interne au linguistique) du concept (entité externe au linguistique), et donc de travailler avec quatre entités. J'avoue mon hésitation. Nous 
sommes devant le problème de choisir entre la tradition et Saussure d'un côté, et une autre conception déjà apparue au XIVe siècle avec la théorie de Grégoire de Rimini. Ockham, à la suite de Boèce et de saint Augustin, avait postulé l'existence d'un langage mental (cf. Boisson, Basset et Kirtchuk 1994). Mais Grégoire n'envisageait pas seulement un seul type de propositiones mentales, mais deux, et c'est, à ma connaissance, le premier à avoir opéré explicitement ce dédoublement:

Appealing to Augustine and Anselm, Gregory («Prologus», q. 1, art. 3) distinguishes, apart from the written and vocal "propositio", two kinds of mental "propositio". One kind consists of mental images of vocal (or written) "propositiones" ; such mental "propositiones" belong to a particular language and are as diverse as the vocal utterances of which they are the likenesses. The other kind is formed by the mental "propositiones" in the proper sense which do not belong to any particular language and are the natural signs that precede the written marks, the vocal sounds, or the mental images. (Nuchelmans 1973: 227-228; aussi Biard 1989: 271)

Six siècles plus tard, on retrouve chez Pottier (1992a : 66, 67; cf. aussi, bien plus cursivement, Pottier 1992b) une distinction entre le «sème», «ou trait de contenu linguistique, lié à une langue particulière», et le «noème», «trait de sens posé indépendamment de toute langue naturelle» (le terme noem / noeme a été créé en anglais avant 1866, mais avec un autre sens: voir $O E D$; chez Pottier, il a pu avoir été emprunté à Husserl: cf. Desclés 1993). Pottier développe toute une partie de son ouvrage sur «le conceptuel et le linguistique». Si l'on regarde de près la longue citation donnée plus bas, on verra pourtant qu'il subsiste une certaine ambiguïté quant au statut exact du «noème»: entre-t-il dans le champ de la linguistique (des signifiés), ou dans le champ de la psychologie (des concepts)? Et cela malgré le fait que Pottier dise (p. 69) : «Un 'concept' sera une combinaison de noèmes».

Mon hésitation sur la question de savoir laquelle est la bonne de la théorie Chrysippe-Saussure ou de la théorie Grégoire de Rimini-Pottier est liée sans doute au fait que si, en tant que linguiste, je suis attaché à l'appréciation en propre des entités linguistiques, par ailleurs je refuse de me laisser emporter par les puissantes tendances réductionnistes panlinguistiques qui soufflent dans l'air de ce temps. C'est tout le statut de la linguistique qui est en cause ici. Faut-il englober les entités mentales traitées par la linguistique dans les entitées mentales traitées par la psychologie, en considérant que, objectivement, ce sont les mêmes, mais que, par simple commodité académique, on en fournira des traitements séparés dans deux disciplines qui ont des axes de recherche quelque peu différents? Ce serait la solution vers laquelle je pencherais, sans certitude.

Par ailleurs, bien entendu, il faut bien voir la différence entre les unités auxquelles j'ai abouti dans l'analyse des termes désignant la règle à calcul dans différentes langues et ce que peut donner une analyse sémique, qui est l'aboutissement d'une procédure différente. En effet, typiquement, les sèmes se dégagent par opposition à l'intérieur d'une seule langue entre plusieurs termes d'un champ sémantique donné, comme dans l'analyse faite par Pottier des sièges. Or, si l'on élabore l'analyse sémique de règle à calcul en contrastant la règle à calcul avec d'autres calculateurs, on va se trouver devant un résultat différent de mon analyse des termes, et ceci d'autant que le champ (sémantique ??? n'estce pas plutôt technologique?) des calculateurs variera suivant l'époque. À la fin du XVII ${ }^{\mathrm{e}}$ siècle, on aura par exemple: règle à calcul / bâtons de Neper / compas de proportion / tables numériques (plus pascaline / machine de Leibniz, mais ces calculateurs n'étaient que des prototypes); vers 1930, on aura: règle à calcul / machine à calculer mécanique / tables numériques; vers 1970 : règle à calcul / calculatrice électronique / ordinateur. 
Mais justement, ce problème de comparaison peut se révéler intéressant en luimême. Il était impossible à percevoir tant que l'on étudiait les sièges, car en général, les termes les désignant sont opaques et indécomposables, si ce n'est par étymologie, en sorte que la simple inspection de chacun des signifiants dans diverses langues ne pouvait en général rien livrer sur le signifié (et éventuellement le concept). En revanche, dans le cas de termes composés, comme sont tous les termes désignant la règle à calcul dans toutes les langues que j'ai étudiées, il y a en surface la projection physique immédiatement «lisible»d'une représentation sémantique complexe. On peut même atteindre ceci partiellement dans le cas des sièges, sinon pour tabouret, chaise, et banc, du moins pour ces termes: (a) les termes pour fauteuil en allemand (Lehnstuhl = «chaise à appuis»), en anglais et en espéranto (armchair, brakse $\hat{g} o=\langle$ chaise à bras»), voire en italien (poltrona = «la paresseuse») et en espagnol (sillón, sur silla, «chaise», avec le suffixe augmentatif), et (b) les termes allemands pour canapé (Polsterbank $=$ «banc à coussins»; Lehnbank $=$ «banc à appuis»).

Il y aurait donc les procédures suivantes pour une analyse sémantique: (1) L'analyse de l'objet pris en lui seul, qui est la méthode lexicographique traditionnelle, mais qui opère sans suffisamment de conditions contraignantes. (2) La méthode étymologique, dans les faits quasiment inutile, voire inductrice d'erreurs, comme on le voit dans fauteuil, qui s'étymologise «siège pliant», ce qui ne correspond plus à rien. (3) Deux types de méthodes comparatives: (a) une comparaison intra-langue, en plongeant le terme dans son champ sémantique (à supposer qu'il n'y en a qu'un, pour simplifier); (b) une comparaison inter-langues, en plongeant le terme dans l'ensemble des désignations de l'objet considéré. Cette dernière procédure d'analyse sémantique proposée par Thoiron semble nouvelle, mais a été préfigurée dans son principe par Leibniz, dans la citation mise en exergue de cet article. Son défaut est évidemment que la finesse d'analyse dépend de l'échantillon de langues retenues, comme on l'a vu; et même il y a avantage à rassembler un corpus assez vaste pour voir se dessiner des conclusions autres que grossières.

Cette prise en compte d'une procédure comparative me conduit à citer assez longuement Pottier :

La distinction fondamentale entre sème et noème est la suivante:

- le sème est le trait distinctif sémantique d'un sémème, relativement à un petit ensemble de termes réellement disponibles et vraisemblablement utilisables chez le locuteur dans une circonstance donnée de communication;

- le noème est un trait de sens posé indépendamment de toute langue naturelle. Il est absolu (et non relatif à un ensemble) et son existence est décidée par l'analyste. Naturellement, le linguiste a créé les noèmes sur la base d'une certaine expérience qu'il a eue des langues naturelles. Mais il ne les tire pas automatiquement des sèmes génériques (ou de classe), bien que l'affinité soit évidente.

Dans l'ensemble \{jument / cheval\} disponible chez un enfant de classe primaire, le sème / femelle / est distinctif par son opposition au sème / mâle /; dans l'ensemble \{auto / moto / vélo , le sème / 4 roues / l'est également, en face de / 2 roues /, et ainsi de suite. On dira d'autre part que le 'sexe', illustré linguistiquement par / mâle / et / femelle /, sera un noème (nécessité de vie universelle), alors que le nombre de roues restera lié à un type de civilisation, à une culture. Ainsi, les 'quelques catégories classématiques majeures du français' données par Anne Hénault sont-elles pratiquement des noèmes.

La noémique est l'étude de l'ensemble des éléments conceptuels et de leurs relations, considérée comme un instrument d'analyse nécessaire pour décrire le fonctionnement de base de la sémantique des langues naturelles. (Pottier 1992a: 67-68) 
Ce qui, pour mon propos local, me trouble dans ce passage de Pottier, c'est l'affirmation selon laquelle le noème «est absolu (et non relatif à un ensemble)». Dès lors, par quelle procédure les noèmes sont-ils dégagés? Et, au contraire, Pottier ne dit-il pas juste après: «Naturellement, le linguiste a créé les noèmes sur la base d'une certaine expérience qu'il a eue des langues naturelles»? Il y a donc comparaison inter-langues, même limitée, informelle et ébauchée.

J'ai considéré les règles d'inférences nécessaires à l'interprétation des termes comme faisant partie du cadre interprétatif associé à ces termes. Le problème est sans doute plus compliqué et doit être repris avec soin. Il est certain en tout cas que les concepts associés aux termes doivent pouvoir jouer toutes sortes de «rôles»: on en trouvera une liste déjà assez longue dans un ouvrage récent (Thagard 1992: 22). Prenant l'exemple du concept BALEINE, Thagard indique que ce concept permet entre autres: (1) la catégorisation (nous pouvons reconnaître certains objets comme des exemples de baleines); (2) l'inférence déductive (puisque Moby Dick est une baleine, elle a des nageoires); (3) l'explication (Moby Dick nage parce qu'elle est une baleine), etc. On retrouve pour le minimum nos inférences.

Nous avons donc ici une vision opérationnelle du concept, le concept n'étant pas un objet à contempler en soi, mais, vu ses relations avec d'autres concepts, une action, une «machine logique» productive (pour une ébauche d'une approche médiévale du concept comme acte de comprendre chez Roger Bacon et chez Walter Chatton, voir Biard 1989: 106-108, 187). Ceci montre donc qu'il est inséré dans ce que l'on pourrait appeler un réseau de fonctions épistémiques, ce qui, notons-le, ne le rend en rien différent de la «valeur» saussurienne, censée être une originalité purement linguistique.

Mon point de vue est évidemment différent, puisque je m'intéresse aux termes, donc à la manière dont ces termes sont compréhensibles, interprétables, à la manière dont le concept est atteignable à partir de sa dénomination.

\section{APPENDICE I : DESCRIPTIONS ET DÉFINITIONS DE LA RÈGLE À CALCUL}

Le Quillet, qui consacre un long article à la règle à calcul système Mannheim, le décrit ainsi, avant d'entrer dans des détails sur la graduation des échelles : «La règle à calcul se compose : $1^{\circ}$ d'une partie fixe avec rainure appelée 'règle'; $2^{\circ}$ d'une partic mobile glissant dans la 'glissière' (ou 'coulisse') de la règle appelée 'réglette' ou encore 'coulisseau', ou 'tiroir'; $3^{\circ}$ d'un verre serti dans un petit cadre métallique: le 'curseur'».

La Chamber's Enyclopedia dit ceci:

Two or more parallel rulers set side by side and allowed relative movement in the direction of their lengths. The slide rule linearly adds together the physical lengths of adjoining segments on adjacent rulers, the appropriate segments being set end to end by sliding the rulers to suitable positions. The graduated scales which delineate these segments are however not linear but logarithmic [...]. With the idea of obtaining high accuracy from a compact instrument slide rules have been made in which the scales (several feet in length) are wrapped spirally round a cylinder.

Voici quelques définitions de dictionnaires divers. L'Oxford English Dictionary définit ainsi l'instrument:

A mathematical gauging or measuring instrument consisting of two graduated parts, one of which slides upon the other, and so arranged that when brought into proper juxtaposition the required result may be obtained by inspection.

Noter que l'OED ne mentionne pas le fait que la graduation est logarithmique! C'est pourquoi le supplément paru en 1986 remplace ceci par la définition suivante: 
A device whereby multiplication and division, and sometimes other mathematical operations, may be performed with speed but limited accuracy, consisting essentially of two rules marked with logarithmic scales and capable of being slid along one another, and usually also a transparent cursor marked with a line crossing the scales, so that a required result may be obtained by inspection after proper movement of these.

L'amélioration est spectaculaire et peut s'expliquer en partie par le plus grand intérêt pour la technique en lexicographie actuelle, et en partie par le fait qu'au moment de la parution du supplément l'instrument cessait d'être employé, risquait de devenir moins familier au lecteur et qu'ainsi une caractérisation rigoureuse devenait d'autant plus requise - on sait que souvent les dictionnaires sont allusifs et que leurs définitions ne se comprennent que dans un «cadre interprétatif» d'informations familières.

Le Webster's Third New International Dictionary offre cette définition (judicieusement accompagnée d'un dessin):

An instrument consisting in its simple form of a ruler and a medial slide that are graduated with similar logarithmic scales labeled with the corresponding antilogarithms and used for rapid calculation.

Le dictionnaire Random House donne cette définition:

A device for rapid calculation, consisting essentially of a rule having a sliding section running along its middle, marked like the rule itself with graduated, usually logarithmic scales.

Barnhart (1974):

A rule with a sliding section in the center, both marked with logarithmic scales, used by engineers, physicists, etc., for making rapid calculations.

Funk \& Wagnalls (1962):

A device consisting of a rigid ruler with a central sliding piece, both ruler and slide being graduated in a similar logarithmic scale to permit rapid calculations.

Dorian (1979):

Simple calculator, consisting of fixed and sliding scales logarithmically graduated.

Parker (1989):

A mechanical device, composed of a ruler with sliding insert, marked with various number scales, which facilitates such calculations as divisions, multiplication, finding roots, and finding logarithms.

\section{Seifert (1977):}

A mechanical analog computing aid which is used extensively for multiplication and division and to a lesser degree for looking up functions. In its most common form a slide rule consists of a body formed from two parallel members rigidly fastened together, a slide which can be moved left or right between the body members, and a transparent indicator which carries a hairline and can be moved left or right over the face of the body and the slide. Scales are provided on the body and the slide as shown in the illustration.

Puis viennent des explications sur les échelles, etc. Noter le renvoi explicite à l'illustration. Le Littré: 
Règle à coulisse marquée de divisions et de chiffres, à l'aide de laquelle on fait très rapidement les calculs. On dit aussi règle logarithmique parce que les résultats des opérations se trouvent par les logarithmes.

\section{Le Petit Robert:}

Instrument composé de deux règles à graduation logarithmique, coulissant l'une sur l'autre, et qui permet d'effectuer rapidement certaines opérations.

\section{Le Grand Larousse de la Langue Française (1986):}

Règle dans laquelle coulisse une réglette, toutes deux portant une graduation logarithmique, et qui permet d'effectuer divers calculs rapides.

\section{Le Trésor de la Langue Française (1990):}

Instrument formé de deux règles coulissant l'une sur l'autre et d'un curseur (ou réglette) qui permet, grâce à des graduations logarithmiques d'effectuer rapidement des opérations mathématiques.

On observera que «coulissant l'une sur l'autre» n'est pas très clair, et trahit quelque peu la réalité. L'Encyclopédie des Sciences Industrielles Quillet (1973: 77):

La propriété du groupe additif $\mathbf{R}$ dans le groupe multiplicatif $\mathbf{R}+$ de l'exponentielle est à la base d'un instrument de calcul usuel : la règle à calcul, qui permet de faire rapidement des multiplications approchées.

Ici le principe de l'instrument est donné sous sa forme la plus abstraite et la plus «moderne», peut-être pas nécessairement la plus éclairante; on a l'impression d'une espèce de raffinement byzantin au moment même où l'objet ainsi ensemblistement exalté va disparaître.

\section{APPENDICE II : OUVERTURES POSSIBLES SUR D'AUTRES DONNÉES}

Il vaudrait sans doute la peine d'étendre l'étude par exemple sur des instruments ou dispositifs à partie coulissante, comme le vénérable trusquin (= anglais marking gauge = italien trusquino / graffietto), ou encore des objets comme:

- valve tiroir / soupape à tiroir $=$ anglais slide-valve $=$ allemand Schieberventil

- banc à glissière $/$ à coulisse $=$ anglais sliding seat $($ aviron $)=$ allemand Rollsitz

— trombone à coulisse $=$ anglais slide-trombone $=$ italien trombone a tiro

- panneau mobile $=$ anglais sliding panel

- toit ouvrant $=$ italien tetto apribile $=$ anglais sliding roof $=$ allemand Schiebedach .

On voit émerger les notions de généralité variable COULISSER, ROULER, OUVRIR, TIROIR, MOBILE.

Mais particulièrement intéressant est le pied à coulisse :

1) Français pied à coulisse / calibre à coulisse / jauge à coulisse / compas à coulisse, employé aussi par les cordonniers (ainsi dans le Grand Dictionnaire Universel de Pierre Larousse) / équerre à coulisse

2) Anglais caliper rule / caliper gauge / slide caliper / sliding gauge / caliper square / vernier caliper (les dénominations semblent foisonnantes)

3) Allemand Schublehre («pousser» + «mesure / calibre») et aussi Schib(e)lehre, Meßschieber

4) Néerlandais schuifmaat, schuifpasser, kaliberpasser 
5) Espagnol calibrador de corredera, calibrador de cursor, calibre corredizo, calibre de colisa; un dictionnaire donne aussi pie de rey, qui doit être au départ une unité de longueur, le «pied de roy», puis un étalon de cette unité, et des règles à charnière en deux pièces pour la mesure (voir par exemple Velter et Lamothe 1977 : 205, 341)

6) Italien calibro a corsoio

7) Hébreu mad Sovi, sur mad = instrument de mesure, et Sovi = épaisseur; si c'est pour le diamètre interne (calibre), mad qoter, où qoter = diamètre (information de Pablo Kirtchuk).

Ce dernier instrument est intéressant à comparer à la règle à calcul en tant que ce que j'appellerai système coulissant gradué. Les systèmes coulissants gradués ont soit une fonction de calcul (règle à calcul), soit une fonction de mesure (s'opposant à ce qu'on pourrait appeler le système à vis gradué du micromètre ou palmer, anglais micrometer caliper, inventé au XVII siècle par Auzout; ou le système encore différent de la clef $\grave{a}$ molette ou clef à crémaillère).

Il est beaucoup plus difficile de trouver des renseignements sur l'histoire du pied à coulisse. Voici le peu que j'en sais. Le pied à coulisse, dans son principe simple, est connu en menuiserie dès le XVI ${ }^{\mathrm{e}}$ siècle (Feller \& Tourret 1970; Gille 1978b: 632). Il s'agit d'un ensemble avec deux becs, l'un solidaire d'une règle graduée, l'autre d'une partie coulissante; les deux becs étant en somme les avatars techniques des branches d'un compas (à mesurer). L'amélioration due à Pierre Vernier en 1631 consiste en la possibilité de mesures linéaires fines grâce au vernier (ensemble de deux règles coulissantes inégalement graduées).

On observera un phénomène que nous avions déjà noté dans le cas de la règle à calcul, à savoir que le pied à coulisse ne semble pas appeler des dénominations par rangement sous une désignation fonctionnelle générique (instrument de mesure), telle que ou *pied à mesure, *pied mesureur, ou *bec à mesure, *mâchoires à mesure ou, par renversement de l'orientation thématique, *jauge à pieds, etc. Ceci sauf dans le cas de l'allemand Schublehre, très approximativement glosable comme «mesureur à coulisse» (plus précisément, l'idée est de «pousser», mais elle rend «coulisser»), et dans le cas de l'hébreu, isolé dans le petit échantillon.

À titre d'exercice de terminologie-fiction, il est intéressant de rechercher des dénominations possibles pour un objet, telles que celles que j'ai étoilées, et qui auraient (en principe) pu exister, et même de se demander pourquoi elles ne sont pas apparues (à moins qu'elles se rencontrent dans tel document inconnu de moi).

Ces études sont aisément extensibles à d'autres instruments ou machines, ainsi l'abaque chinoise, appelée suan phan (calculating plate: dénomination hétérogène morpho-fonctionnelle) ou bien chu suan (ball plate: dénomination homogène uniquement fonctionnelle). Ou encore, songer à la bicyclette et à ses ancêtres divers, à propos de laquelle on peut facilement énumérer dans plusieurs langues les termes morphologiques (y compris métaphoriques), fonctionnels ou éponymiques: céléripède, célérifère, vélocipède (resté dans le russe velosipéd), vélocifère, Laufmaschine (nom nommé à la draisienne par Drais), draisienne, michauline, penny-farthing, puis bicycle, bicyclette, Zweirad ou Fahrad, le terme chinois fait à neuf zi xíng che et qui se glose «individueldéplacement-véhicule» (Pottier 1992b: 45).

Enfin, et naturellement, la caractéristique de toute dénomination (la sélection de telle ou telle parmi les diverses caractéristiques d'une entité) ne vaut pas seulement pour les artefacts, mais aussi, dans une certaine mesure, pour les animaux et les plantes: voir ainsi serpent à sonnette (= anglais rattlesnake, et l'espéranto, à premier élément plus général, sonserpento), le fréquent duckbilled platypus, redondant à côté du simple 
platypus, etc., termes où une caractéristique saillante a été sélectionnée, en négligeant les autres.

\section{RÉFÉRENCES}

Pour les traductions, on ne citera pas ici en général les dictionnaires généraux de langue et les dictionnaires spécialisés utilisés.

AGAZZI, E. (1978): «Les critères sémantiques pour la contitution de 1'objet scientifique», M. Bunge et alii (dir.), La Sémantique dans les sciences, Colloque de l'Académie internationale de philosophie des sciences, Paris, Beauchesne, pp. 13-54.

ANONYME (1825): Instruction sur la manière de se servir de la règle à calcul, instrument à l'aide duquel on peut obtenir à vue, sans plume, crayon ni papier, sans barrême, sans compte de tête, et même sans savoir l'arithmétique, le résultat de toute espèce de Calculs, Paris, Bachelier / Dijon, Douillier.

ARNAUD, P. (1987): Aspects du vocabulaire et de son évaluation lors de l'acquisition d"une langue étrangère (anglais), Thèse de Doctorat d'État, Université Paris 7 (Microfiches).

ARTUR, J.-F. (1827): Instruction théorique et applications de la règle logarithmique ou à calculs, Paris, Carilian-Goeury.

ASIMOV, I. (1972) : Asimov's Biographical Encyclopedia of Science and Technology, new revised edition, New York, Doubleday \& Company.

AUBERT (dir.) (1991) : «Mesures et contrôles», Techniques de l'ingénieur, $R$ 4, Paris.

BARNHART, C. L. (Ed.) (1974): The World Book Dictionary, Thorndike Barnhart.

BAXANDALL, D. (1926): Catalogue of the Collections in the Science Museum, South Kensington, With descriptive and historical notes and illustrations. Mathematics I. Calculating machines and instruments, Londres, His Majesty's Stationery Office.

BAXANDALL, D. (1929): «Calculating machines», Encyclopcedia Britannica, 14e édition.

BELAVAL, Y. $(1952,1989)$ : Leibniz, initiation à sa philosophie, Paris, Vrin.

BIARD, J. (1989): Logique et théorie du signe au XIVe siècle, Paris, Librairie philosophique J. Vrin.

BLAZ̆JEK, V. et C. BOISSON (1992): «The Diffusion of Agricultural Terms from Mesopotamia», Archiv Orientální, $\mathrm{n}^{\circ} 60, \mathrm{pp} .16-37$.

BOISSON, C. (1985): «La métaphore en linguistique et en psychologie cognitive», S/GMA, n ${ }^{\circ}$ 9, pp. 27-55.

BOISSON, C. (1991): «La place de l'image mentale en sémantique», RANAM, $\mathrm{n}^{\circ} 23$, pp. 1-12.

BOISSON, C., KIRTCHUK, P. et H. BÉJOINT (1991): «Aux origines de la lexicographie: les premiers dictionnaires monolingues et bilingues», International Journal of Lexicography, $\mathrm{n}^{\circ}$ 4, pp. 261-315.

BOISSON, C., BASSET, L. et P. KIRTCHUK (1994): «Problématiques des parties du discours», L. Basset et M. Pérennec (dir.), Classes de mots: traditions et perspectives, Lyon, Presses Universitaires de Lyon, pp. 9-45.

BRYDEN, D. J. (1992): «Evidence from Advertising for Mathematical Instrument Making in London, 1556-1714», Annals of Science, $\mathrm{n}^{\circ} 49, \mathrm{pp} .301-336$.

CAJORI, F. (1909): A History of the Logarithmic Slide Rule. [Cité dans Baxandall; je n'ai pu le consulter.]

Chamber's Encyclopedia (1955): Londres.

CHARPENTIER, P. et H. LAURENT (1885-1902): «Arithmomètre», La Grande Encyclopédie, Paris.

COLLARDEAU (1833): Instruction sur l'usage de la règle à calculs portative, à une seule coulisse, Paris, Imprimerie de Mme Vve Thuau.

Comment ça marche, Encyclopédie pratique des inventions et des techniques (1980): vol. 8, Bruxelles, Éditions Atlen / Lugano, Éditions transalpines.

DASCAL, M. (1978) : La sémiologie de Leibniz, Paris, Aubier Montaigne.

DAUMAS, M. (1953) : Les Instruments scientifiques aux XVII et XVIII siècles, Paris, Presses Universitaires de France.

DEMORIANE, H. (1974) : L'Art de reconnaître les instruments scientifiques du temps passé, Paris, Hachette.

DESCLÉS, J.-P. (1993) : Primitives sémantico-cognitives pour la représentation de la signification des verbes de mouvement en français, Conférence à la Maison Rhône-Alpes des Sciences de l'Homme, 8 janvier 1993.

DE VIRVILLE, M. et alii (1977): Système descriptif des objets domestiques français, Centre d'ethnologie française, Musée national des arts et traditions populaires, Paris, Éditions des musées nationaux, Paris.

DHOMBRES, J. et alii (dir.) (1987): Mathématiques au fil des âges, Textes choisis et commentés, Paris, Gauthier-Villars.

Dictionnaire encyclopédique Quillet (1953): Paris, Aristide Quillet.

DIDEROT, D. et J. D'ALEMBERT (dir.) $(1753,1777)$ : Encyclopédie ou dictionnaire raisonné des sciences, des arts et des métiers, Paris.

D'OCAGNE, M. (1905): Le Calcul simplifié par les procédés mécaniques et graphiques. Histoire et description sommaire des instruments et machines à calculer, tables, abaques et nomogrammes, Paris, Gauthier-Villars.

DORIAN, A. F. (1979): Dictionary of Science and Technology, English-French, Amsterdam, Elsevier.

Encyclopeedia Britannica (1974): Chicago. 
Encyclopédie des Sciences Industrielles Quillet, Électricité-Electronique-Génêralités (1973): Paris, Librairie Aristide Quillet.

Everyman's Encylopadia (1949-1950): 3rd edition, Londres, Dent.

FELLER, P. et F. TOURRET (1970): L'Outil, dialogue de l'homme avec la matière, Rhode-St-Genèse (Belgique), Albert De Visscher.

FLEGG, G. (1983): Numbers, Their History and Meaning, New York, Schocken Books.

Funk \& Wagnalls Standard Dictionary of the English Language, International Edition (1962): Chicago, Encyclopredia Britannica / New York, Funk \& Wagnalls.

GILLE, B. (1978a): «Prolégomènes à une histoire des techniques», B. Gille (dir.), Histoire des techniques, Paris, Gallimard, pp. 1-118.

GILLE, B. (1978b): «Les systèmes classiques», B. Gille (dir.), Histoire des techniques, Paris, Gallimard, pp. 580-676.

GILLE, B. (dir.) (1978) : Histoire des techniques, Collection «Encyclopédie de la Pléiade», Paris, Gallimard.

GILLISPIE, C. C. (Ed.) (1970-1976): Dictionary of Scientific Biography, 14 volumes, New York, Charles Scribner's Sons.

Grande enciclopedia portuguesa e brasileira, Lisboa / Rio de Janeiro, Editorial Enciclopédia

GUIRAUD, P. (1978): Les Mots savants, Paris, Presses Universitaires de France.

HAIMAN, J. (1980) : «Dictionaries and Encyclopedias», Lingua, ${ }^{\circ}$ 50, pp. 329-357.

HOTTOIS, G. (1989): Penser la logique, Une introduction technique, théorique et philosophique à la logique formelle, Bruxelles, De Boeck.

HUXLEY, G. (1970): «Henry Briggs», Gillispie (Ed.), Dictionary of Scientific Biography, vol. 2, New York, Charles Scribner's Sons, pp. 1561-1630.

ITARD, J. (1972): «Denis / Didier Henrion», Gillispie (Ed.), Dictionary of Scientific Biography, vol. 6, New York, Charles Scribner's Sons, pp. 271-272.

JANTON, P. (1977): L'Espéranto, $2^{\mathrm{e}}$ édition, Coll. «Que sais-je?», Paris, Presses Universitaires de France.

KALOCSAY, K. et G. WARINGHIEN (1985) : Plena analiza gramatiko de Esperanto, Kvina korektita eldono, Rotterdam, Universala Esperanto-Asocio.

KETTRIDGE, J. O. (1980): French-English and English-French Dictionary of Technical Terms, Vol. 1, Londres, Routledge \& Kegan Paul.

KOPPELMAN, E. (1974): «Amédée Mannheim», Gillispie (Ed.), Dictionary of Scientific Biography, vol. 9, New York, Charles Scribner's Sons.

LAMI, E.-O. (dir.) (1882): Dictionnaire encyclopédique et biographique de l'industrie et des arts industriels, Paris, Librairie des Dictionnaires.

LEIBNIZ, G. W. (1765, 1990) : Nouveaux essais sur l'entendement humain, Paris, GF-Flammarion.

LE NY, J.-F. (1979): La Sémantique psychologique, Paris, Presses Universitaires de France.

LIGONNIĖRE, R. (1987): Préhistoire et histoire des ordinateurs, Paris, Robert Laffont.

MARGUIN, J. et alii (1990): De la machine à calculer de Pascal à l'ordinateur, 350 ans d'informatique, Exposition du 26 avril au 23 septembre 1990, Musée National des Techniques, Conservatoire National des Arts et Metiers, Paris.

MESSADIÉ, G. (1988): Les Grandes inventions de l'humanité jusqu' en 1850, Paris, Bordas.

MICHAUD, (1842-1865): Biographie universelle ancienne et moderne, nouvelle édition, 45 vols., Paris, Desplaces.

MICHEL, H. (1973): Instruments des sciences dans l'art et dans l'histoire, Bruxelles, Albert de Visscher

NEEDHAM, J. et W. LING (1959): Science and Civilisation in China, Volume 3: Mathematics and the Sciences of the Heaven and the Earth, Cambridge, Cambridge University Press.

NOSBAN, M. (1873, 1977): Nouveau manuel complet du menuisier en batiments et du layetier-emballeur, nouvelle édition, Paris, Léonce Laget.

NOVY, L. (1970): «Joost Bürgi», Gillispie (Ed.), Dictionary of Scientific Biography, vol. 2, New York, Charles Scribner's Sons, pp. 602-603.

NUCHELMANS, G. (1973): Theories of the Proposition: Ancient and Medieval Conceptions of the Bearers of Truth and Falsity, Amsterdam / Londres, North-Holland.

OHLMANN, H. (1990): «Information: Timekeeping, Computing, Telecommunications and Audiovisual Technologies», I. McNeil (Ed.), An Encyclopedia of the History of Technology, Londres / New York, Routledge, pp. 686-758.

PARKER, S. P. (Ed.) (1989): McGraw-Hill Dictionary of Scientific and Technical Terms, 4th edition, New York, McGraw-Hill.

PEPPER, J. V. (1972): «Edmund Gunter», Gillispie (Ed.), Dictionary of Scientific Biography, vol. 5, New York, Charles Scribner's Sons, pp. 593-594.

POTTIER, B. (1992a): Théorie et analyse en linguistique, $2^{\mathrm{e}}$ édition, Paris, Hachette.

POTTIER, B. (1992b) : Sémantique générale, Paris, Presses Universitaires de France. 
QUEMADA, B. (1978): «Technique et langage», B. Gille (dir.), Histoire des techniques, collection «Encyclopédie de la Pléiade», Paris, Gallimard, pp. 1146-1240.

The Random House College Dictionary (1975): reviscd edition.

SCOTT, J. F. (1974): «William Oughtred», Gillispie (Ed.), Dictionary of Scientific Biography, vol. 10, New York, Charles Scribner's Sons, pp. 254-255.

SEIFERT, W. W. (1977): «Slide rule», McGraw-Hill Encyclopedia of Science and Technology, 15 volumes, New York, McGraw-Hill.

SELLA, Q. (1859): Teorica e pratica del regolo calcolatore, Stamperia reale, Torino.

SINGER, C. et alii (Eds.) (1957): A History of Technology. Volume III: From the Renaissance to the Industrial Revolution, c. $1500-$ c. 1750, Oxford, Clarendon Press

STEPHEN, L. et S. LEE (Eds.) (1917-): The Dictionary of National Biography, Oxford, Oxford University Press.

TALMY, L. (1985): «Lexicalization patterns», T. Shopen (Ed.), Language Typology and Syntactic Description, vol. 3, Cambridge, Cambridge University Press, pp. 57-149.

THAGARD, P. (1992): Conceptual Revolutions, Princeton, Princeton University.

THOIRON, P. (1994): «La terminologie multilingue: une aide à la maîtrise des concepts», Meta, numéro spécial : Hommage à Bernard Quemada : termes et textes, vol. 39, n 4 , pp. 765-773.

TURNER, A. J. (1973) : «Mathematical instruments and the education of gentlemen», Annals of Science, $n^{\circ} 30$, pp. $51-88$.

TURNER, A. J. (1988): «'Utile pour les calculs' : the logarithmic scale rule in France and England during the seventeenth century», Archives Internationales d'Histoire des Sciences, $n^{\circ} 38$, pp. 252-270

VELTER, A. et M.-J. LAMOTHE (1977) : Le Livre de l'Outil, Éditions Hier et demain.

VOGEL, K. (1976): «Michael Stifel», Gillispie (Ed.), Dictionary of Scientific Biography, vol. 13, New York, Charles Scribner's Sons.

WARINGHIEN, G. (Ed.) (1970): Plena ilustrita vortaro de Esperanto, Paris, Sennacieca Asocio Tutmonda.

WELLS, J. C. (1969, 1992): Concise Esperanto Dictionary and English Dictionary, Collection Teach Yourself Books, Sevenoaks (Kent), Hodder and Stoughton.

WELLS, J. C. (1989): Lingvistikaj aspektoj de Esperanto, Dua eldono, Rotterdam, Universala EsperantoAsocio.

WYNTER, H. et A. TURNER (1975) : Scientific Instruments, Londres, Studio Vista 